\title{
Joint report on terminology for surgical procedures to treat pelvic organ prolapse
}

\author{
Developed by the Joint Writing Group of the American Urogynecologic Society \\ and the International Urogynecological Association
}

Published online: 10 February 2020

(C) American Urogynecologic Society and International Urogynecological Association 2020

\begin{abstract}
Surgeries for pelvic organ prolapse (POP) are common, but standardization of surgical terms is needed to improve the quality of investigation and clinical care around these procedures. The American Urogynecologic Society and the International Urogynecologic Association convened a joint writing group consisting of 5 designees from each society to standardize terminology around common surgical terms in POP repair including the following: sacrocolpopexy (including sacral colpoperineopexy), sacrocervicopexy, uterosacral ligament suspension, sacrospinous ligament fixation, iliococcygeus fixation, uterine preservation prolapse procedures or hysteropexy (including sacrohysteropexy, uterosacral hysteropexy, sacrospinous hysteropexy, anterior abdominal wall hysteropexy, Manchester procedure), anterior prolapse procedures (including anterior vaginal repair, anterior vaginal repair with graft, and paravaginal repair), posterior prolapse procedures (including posterior vaginal repair, posterior vaginal repair with graft, levator plication, and perineal repair), and obliterative prolapse repairs (including colpocleisis with hysterectomy, colpocleisis without hysterectomy, and colpocleisis of the vaginal vault). Each of these terms is clearly defined in this document including the required steps of the procedure, surgical variations, and recommendations for procedural terminology.
\end{abstract}

Keywords vaginal prolapse $\cdot$ pelvic organ prolapse $\cdot$ surgery $\cdot$ repair $\cdot$ terminology $\cdot$ standardization

Although surgeries for pelvic organ prolapse (POP) are common [1], lack of standardized terminology leads to confusion among health providers and patients. This inconsistent termi-

Individual contributors are noted in the Acknowledgment section.

This report is being published concurrently in Female Pelvic Medicine and Reconstructive Surgery and in International Urogynecology Journal. The report is identical except for minor stylistic and spelling differences in keeping with each journal's style. Citations from any of the two journals can be used when citing this article.

Correspondence: Kate V. Meriwether, University of New Mexico Hospital, 2211 Lomas Blvd NE, 4th Flr, Department of Obstetrics \& Gynecology, Albuquerque, NM 87106, USA.

E-mail: meriwet2@salud.unm.edu. nology limits both patient and health care provider understanding and inhibits our ability to perform clinical research $[2,3]$.

Despite the introduction of several standardization documents on terminology for POP and pelvic floor dysfunction [3-6], no such document exists for the surgical procedures to repair POP. Different eras in the history of female pelvic reconstructive surgery practice have seen procedures for POP change dramatically or become largely obsolete. For research to produce meaningful data about specific procedures, standardized and widely accepted terminology must be adopted. Each term for a given procedure must indicate to researchers, clinicians, and learners a specific and reliable minimal set of steps. The aim of this document is to propose a standardized terminology to describe common surgeries for POP. 


\section{Methods}

The American Urogynecologic Society (AUGS) and the International Urogynecologic Association (IUGA) convened a joint writing group consisting of 5 designees from each society with expertise on surgical procedures for POP. The authors performed a literature review of commonly performed POP repair procedures and extracted surgical descriptions. After this, the writing group then engaged in an iterative process of discussion of terms and ultimately selected and agreed upon the relevant surgical terminology proposed in this document.

The aim of the writing group was to produce a clinically useful document that comprehensively defines the terminology for surgical repair of POP. The authors sought to develop specific joint terminology to do the following:

1. Produce preferred terminology for each surgical description and provide examples of both acceptable alternative terms and nonrecommended terminology.

2. Describe the historical context of the surgery.

3. Describe the surgery in a clear and stepwise manner, including detailed illustrations where appropriate.

4. Include specific materials and equipment used in the surgery.

5. Address specific limitations and pitfalls surrounding terminology for each surgery.

6. Combine input from AUGS and IUGA with the assistance of designated referees.

7. Provide clinically meaningful terminology for POP surgeries for common use by educators, learners, researchers, clinicians, physiotherapists, and midlevel healthcare providers.

This document clarifies and recommends standardized terminology and discusses the specific nature of a set of surgical terms (Fig. 1) for POP repair procedures that are commonly in use and/or sufficiently present in the medical literature to warrant definition. This document is not intended to discuss clinical outcomes of POP surgeries, review the evidence base for particular POP surgeries, or recommend a particular POP surgery in an individual situation. Inclusion of a surgical term in this document is not an endorsement of the procedure's value, safety, or availability; the aim of this document is to clarify the definition of the procedure as opposed to clinical judgment regarding its application. However, we have indicated in this document instances where surgical steps for a certain procedure have been specifically adapted for the purpose of improving specific outcomes or avoiding specific complications.

\section{Sacrocolpopexy and Sacrocervicopexy}

Sacrocolpopexy (SCP) is defined as suspension of the vaginal apex to the anterior longitudinal ligament of the sacrum using a graft, with possible incorporation of the graft into the fibromuscular layer of the anterior and/or posterior vaginal walls (Fig. 2). The term sacrocervicopexy (SCerP) is defined as suspension of the uterine cervix to the anterior longitudinal ligament of the sacrum using a bridging graft, with possible incorporation of the graft into the fibromuscular layer of the anterior and/or posterior vaginal walls (Table 1).

As early as 1962, the use of a graft to bridge the vaginal vault to the sacrum was described for the treatment of posthysterectomy prolapse [7]. Before that, in 1957, uterine hysteropexy, where the uterine fundus was attached to the anterior longitudinal ligament with suture, was suggested [8]. Suturing the graft to the anterior longitudinal ligament at the level of S1 to S2 has been recommended to minimize bleeding and to avoid the intervertebral disc and the risk of discitis $[9,10]$. A wide variety of different grafts have been used for SCP in history. At present, Amid type 1, macroporous, monofilament, light-weight polypropylene mesh is the most used in published, highquality studies [11-14]. Early descriptions of graft placement described suturing a single strip of graft from the sacral promontory along the rectovaginal septum to the perineum to distribute the graft attachments over a large surface area [15]. Another study proposed attaching the graft on both the anterior and posterior vagina to improve vaginal support across all compartments, which is now commonly performed [16].

\section{Procedure description}

Equipment The most common routes for SCP and SCerP are abdominal, laparoscopic, and robotic, although vaginal approaches have been described, and equipment used would be consistent with approach (Table 2) [17, 18]. A vaginal retractor or dilator of some type is useful to manipulate and delineate the vagina during the case.

\section{List of steps}

Step 1: The procedure begins by gaining access to the intraabdominal cavity (through an open, laparoscopic, or robotic approach), identifying relevant anatomic landmarks (such as the ureters and rectosigmoid), and retracting the rectosigmoid to the left of the sacrum to expose the peritoneum over the sacral promontory.

Step 2: The retroperitoneal space over the bony sacral promontory is entered and a window overlying the 


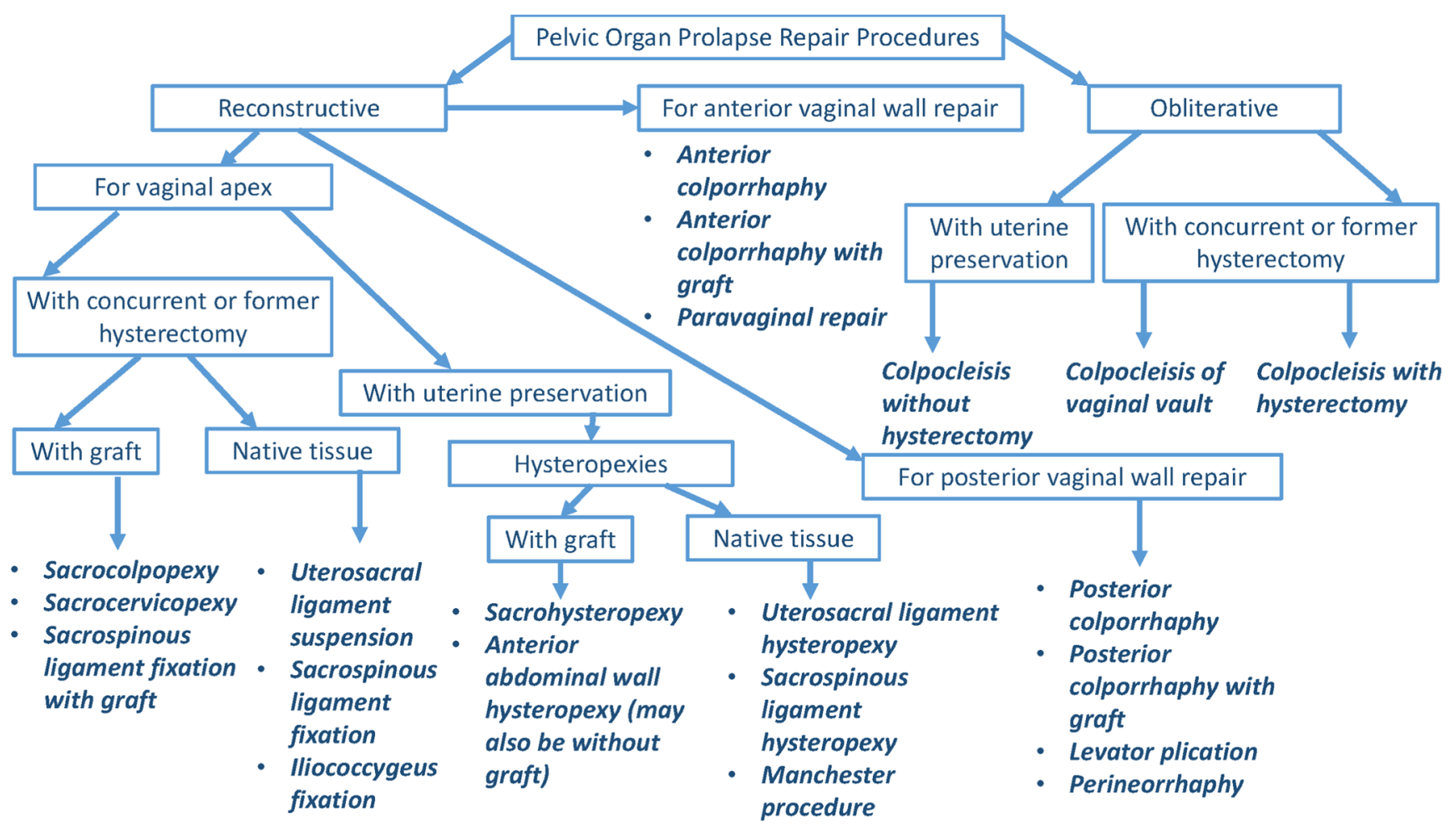

Fig. 1 Flow chart of surgeries for POP by surgery characteristics

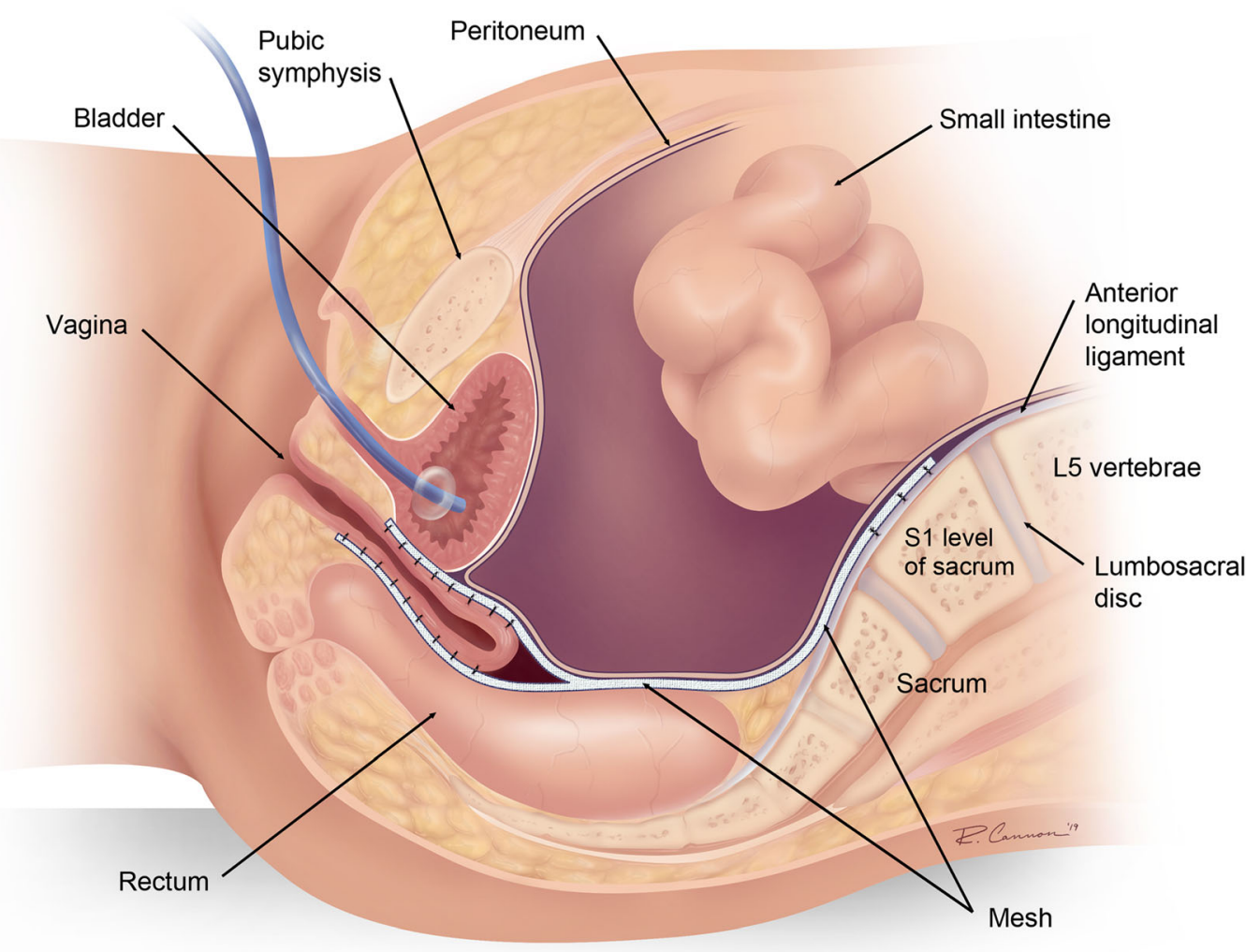

Fig. 2 Sacrocolpopexy 


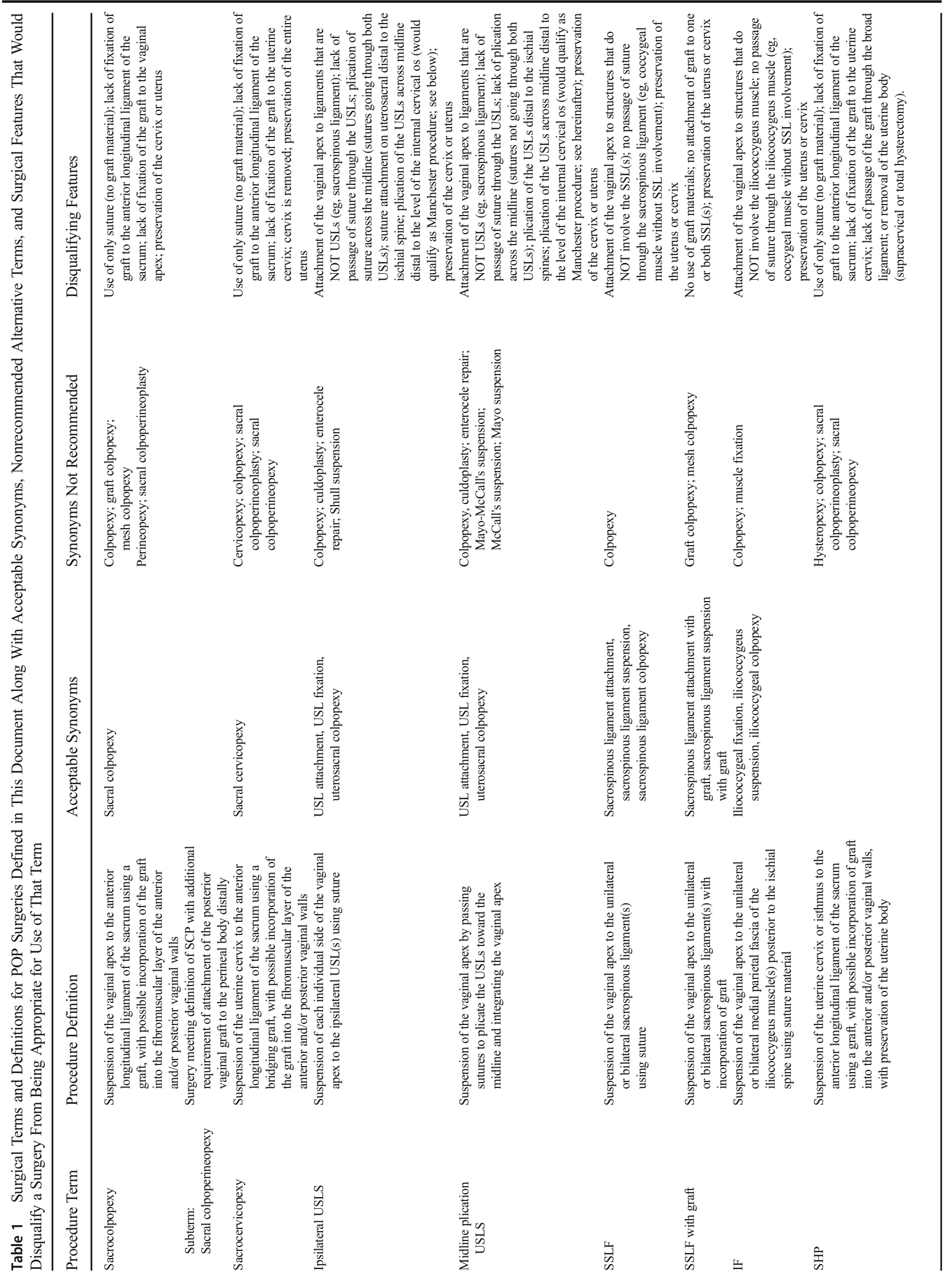




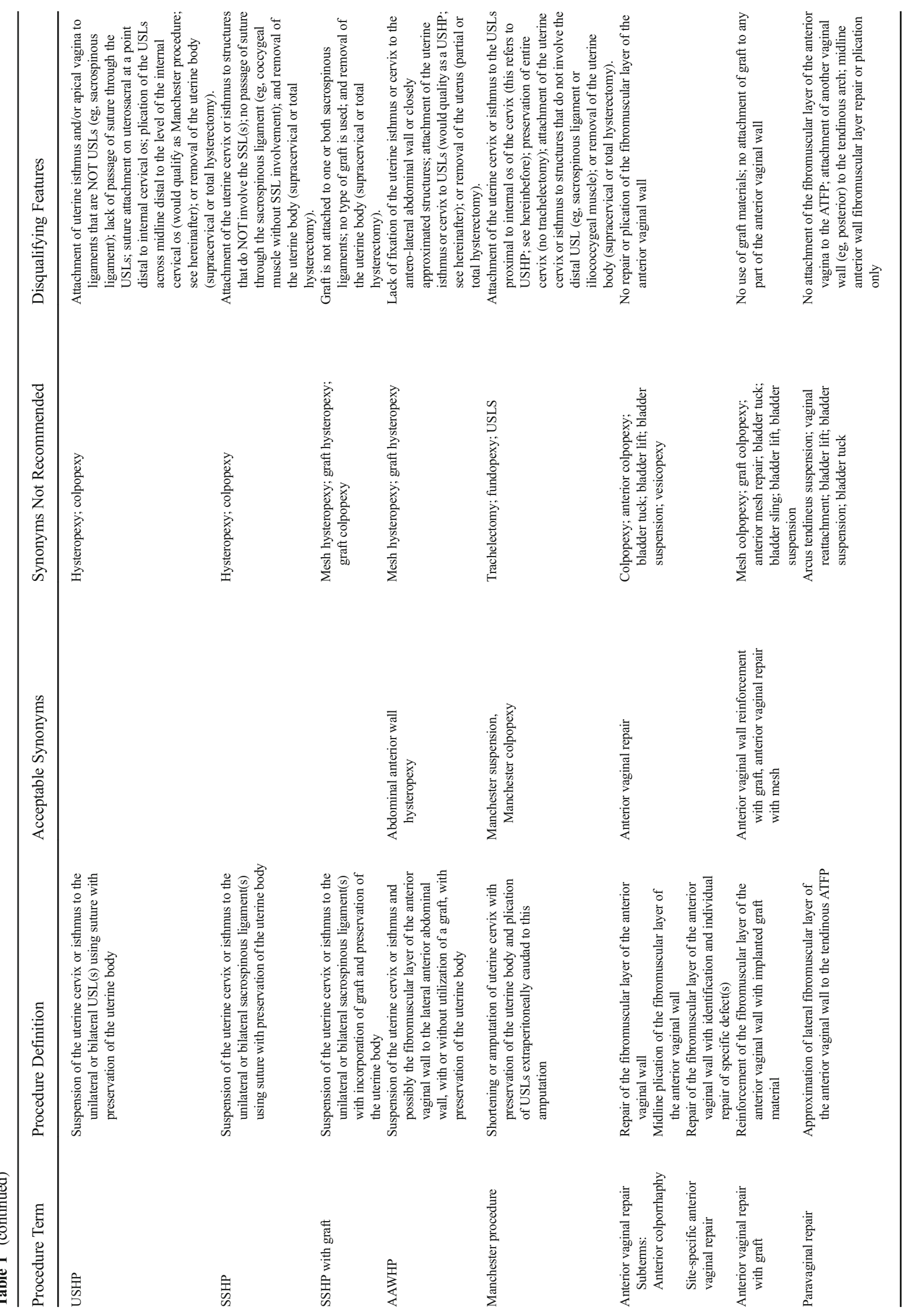



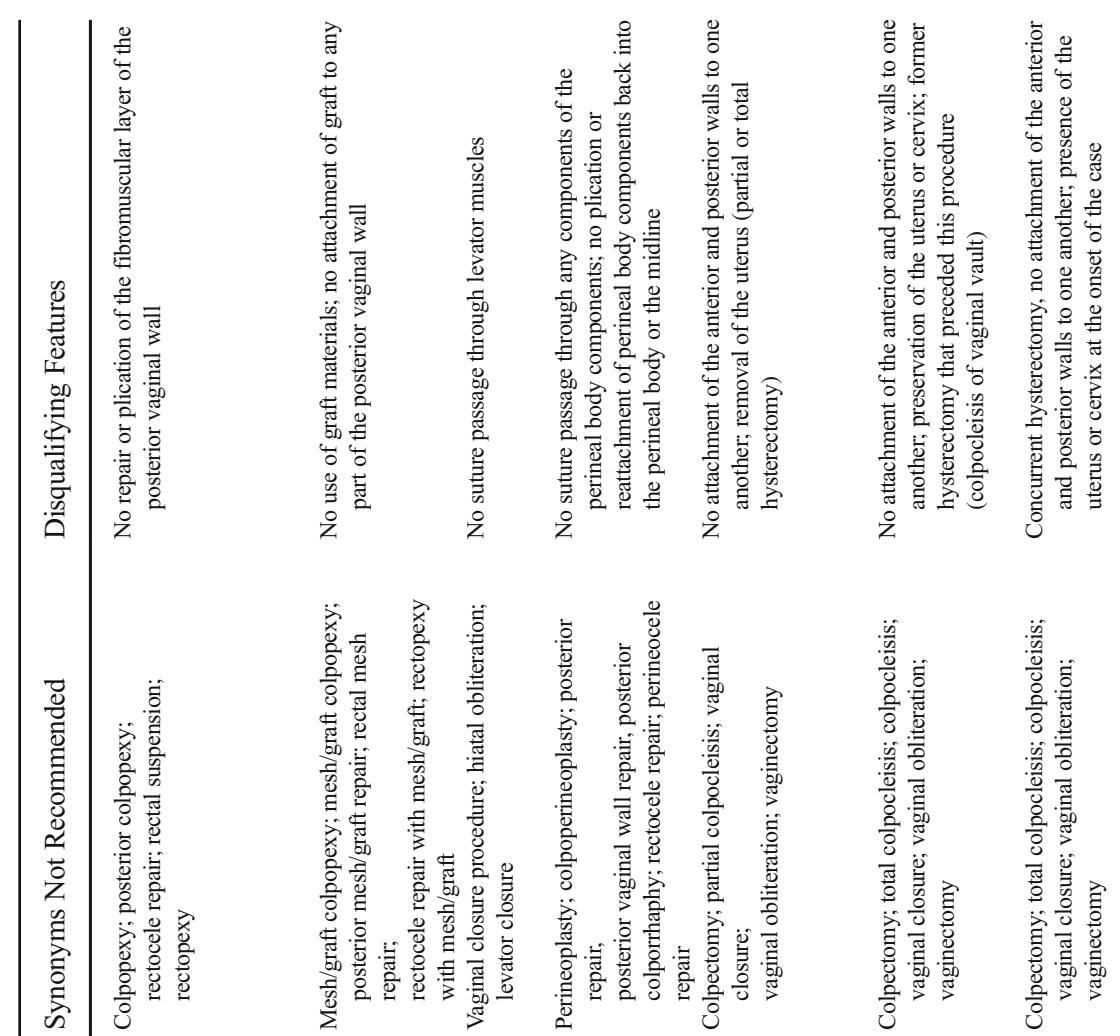

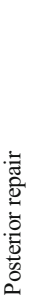
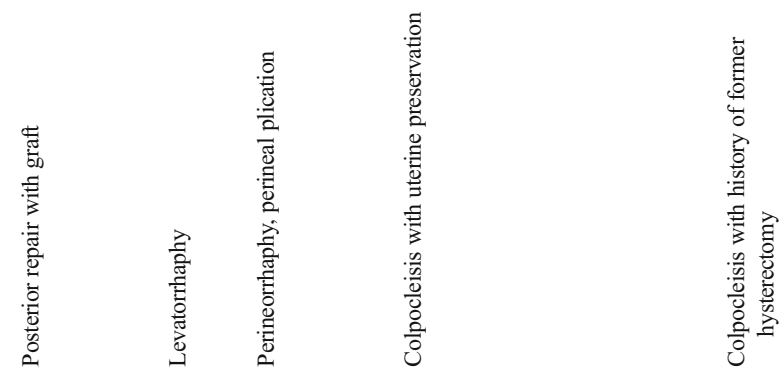

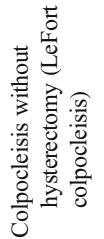

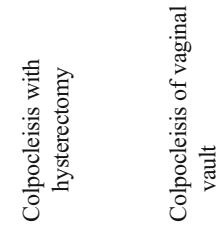


Table 2 Recommended Equipment for POP Surgery by Approach

\section{Laparoscopic Equipment}

Leg stirrups with ability to adjust leg position

Drape with abdominal and vaginal access

Marking pen

Ruler

Blade handle and blade

Vaginal retractors (eg, right angle or Breisky-Navratill)

Foley/urethral catheter

Instrument to manipulate vagina (eg, Lucite rod, vaginal stent, Breisky-Navratrill retractor, EA sizer)

Uterine manipulator

Laparoscopic ports with appropriately sized trocars; with or without visualization trocars

Carbon dioxide tank and gas cord

Veress needle

Laparoscopic camera, light cord, and tower

Laparoscopic lens ( 0 and 30 degrees)

Laparoscopic suction/irrigator

Laparoscopic dissecting instrument with or without energy

Laparoscopic needle drivers

Laparoscopic atraumatic graspers

Laparoscopic Maryland graspers

Laparoscopic scissors

Laparoscopic knot pusher

Hemostatic clamps

Nonabsorbable, monofilament suture

Fascial closure device (eg, Carter Thomason)

Delayed absorbable sutures with small, tapered needles

Small gauge absorbable sutures

Fine, toothed pickups

Cystoscopy setup (70 degree lens, camera and tower, sterile fluid irrigation)

\section{Open Abdominal Equipment}

Leg stirrups with ability to adjust leg position

Drape with abdominal and vaginal access

Marking pen

Ruler

Blade handle and blade

Handheld vaginal retractors (eg, right angle or Breisky-Navratill)

Foley/urethral catheter

Instrument to manipulate vagina (eg, Lucite rod, vaginal stent, Breisky-Navratrill retractor, EA sizer)

Uterine manipulator

Monopolar cautery device

\section{Purpose/Indications}

Vaginal field access and visualization

Sterile field delineation and abdominal/vaginal field separation

Incision planning and landmark mapping on anterior abdominal wall

Measuring of landmarks for incisional planning; assurance

of adequate space between laparoscopic ports

Skin incisions for laparoscopic port sites

Vaginal visualization during manipulator placement; evaluation of vagina for luminal injury; visualization of prolapse reduction during graft tensioning

Delineate bladder margins, decompress bladder to prevent injury, identify urethra

Insert into vagina to delineate vaginal margins and aid in dissection and suturing to abdominal vaginal surface

Manipulation of uterus during concurrent hysterectomy or hysteropexy

Abdominal/peritoneal cavity access

Abdominal insufflation

Optional for peritoneal entry and insufflation

Endoscopic visualization

Straight or angled visualization into pelvic spaces as needed

Field cleaning and irrigation

Dissection of surgical planes, such as development of the vesicovaginal or rectovaginal spaces or dissection of the presacral space

Suturing of graft to abdominal vaginal fibromuscular layers; suturing of graft to anterior longitudinal ligament

Manipulation of bowel or pelvic organs without injury

Fine grasping of peritoneum or other structures

Sharp dissection of surgical planes or cutting of suture material

Securing of knots in extracorporeal tying

Suture handling; blunt dissection

Attachment of graft to anterior longitudinal ligament of sacrum

Optional for suture passage in abdominal fascial closure on port sites $\geq 10 \mathrm{~mm}$

Abdominal fascial closure on port sites $\geq 10 \mathrm{~mm}$

Attachment of graft to fibromuscular layers of vagina; skin incision closure; hemostasis of bleeding sites, repair of visceral organ injury

Skin retraction during skin closure

Examine bladder for injury and ensure ureteral patency at the end of the procedure

\section{Purpose/Indications}

Vaginal field access and visualization

Sterile field delineation and abdominal/vaginal field separation

Incision planning and landmark mapping on anterior abdominal wall

Measuring of landmarks for incisional planning; assurance of adequate space between ports

Skin incision

Vaginal visualization during manipulator placement; evaluation of vagina for luminal injury; visualization of prolapse reduction during graft tensioning

Delineate bladder margins, decompress bladder to prevent injury, identify urethra

Insert into vagina to delineate vaginal margins and aid in dissection and suturing to the abdominal vaginal surface

Manipulation of uterus during concurrent hysterectomy or hysteropexy

Extending incisional dissection; hemostasis; pelvic dissection 
Table 2 (continued)

Suction device with or without irrigation

Self-retaining retractors

Malleable retractors

Laparotomy tapes

Long pickups with and without teeth

Hemostats

Handheld Metzenbaum scissors

Suture scissors

Nonabsorbable, monofilament suture

Delayed absorbable sutures with small, tapered needles

Small gauge absorbable sutures

Fine, toothed pickups

Cystoscopy setup (70 degree lens, camera and

tower,

sterile fluid irrigation)

\section{Vaginal Equipment}

Leg stirrups with or without ability to adjust leg position

Leggings and abdominal drape

Under-buttock drape

Blade handle and blade

Weighted speculum

Handheld vaginal retractors (eg, right angle or

Breisky-Navratill)

Foley/urethral catheter

Monopolar cautery device with or without long tip

Suction device with or without irrigation

Self-retaining retractors

Lighted retractor or head lamp

Tenacula (single toothed or multitoothed)

Long pickups with and without teeth

Hemostats

Allis clamps

Handheld Metzenbaum scissors

Suture scissors

Delayed absorbable sutures with small, tapered needles

Nonabsorbable and/or delayed monofilament sutures

with or without special suture capture devices/

bullets

Suture passage device (eg, Capio® or Deschamps needle driver)

Cystoscopy setup (70 degree lens, camera and tower, sterile fluid irrigation)
Cleaning of field; irrigation

Visceral organ retraction and pelvic visualization

Visceral organ retraction and pelvic visualization

Bowel packing; wound edge and side wall protection; field cleaning

Graft, suture, or tissue manipulation

Suture handling and grasping of bleeding blood vessels

Sharp dissection

Suture cutting

Attachment of graft to anterior longitudinal ligament of sacrum

Abdominal fascial closure; subcutaneous adipose closure if needed

Attachment of graft to fibromuscular layers of vagina; skin incision closure; hemostasis of bleeding sites, repair of visceral organ injury

Skin retraction during skin closure

Examine bladder for injury and ensure ureteral patency at the end of the procedure

\section{Purpose/Indications}

Vaginal field access and visualization

Sterile field delineation and protection

Capture of blood and irrigation fluid; protection against dropping instruments

Vaginal epithelium and/or skin incision(s)

Retraction and protection of rectum and posterior vaginal wall; opening levator ani hiatus Field visualization

Delineate bladder margins; decompress bladder to prevent injury, identify urethra Epithelial incisions; hemostasis

Cleaning of field, irrigation

Labial or vaginal wall retraction and pelvic visualization

Pointed light for improved visualization

Manipulation or traction on cervical stroma if cervix/uterus present

Graft, suture, or tissue manipulation

Suture handling and grasping of bleeding blood vessels

Tissue manipulation and traction

Sharp dissection

Suture cutting

Fibromuscular layer plication; vaginal epithelial closure

Placement through ligament anatomy for apical vaginal suspension (eg, through the uterosacral or sacrospinous ligaments)

Passage of sutures in narrow/long dissected spaces, eg, though the sacrospinous ligament(s)

Examine bladder for injury and ensure ureteral patency at the end of the procedure anterior longitudinal ligament at the level of S1-S2 is developed.

Step 3: The peritoneum is either divided from this dissection along the right pelvic side wall down to the vagina or a retroperitoneal tunnel under the peritoneum is created along the same course, taking care to identify and avoid the nearby ureter and the rectosigmoid. This step is undertaken to retroperitonealize the graft at the end of the procedure, although some surgeons do not perform this step.
Step 4: The bladder is dissected anteriorly and the rectum posteriorly from the vaginal walls.

Step 5: The graft of choice is introduced into the abdomen.

Step 6: The anterior arm is sutured to the anterior vaginal wall and the posterior arm to the posterior vaginal wall using sutures.

Step 7: The graft material is sutured or tacked to the anterior longitudinal ligament to obtain a suspension bridge between the vagina and the sacrum. 
Step 8: Peritoneal closure over the exposed graft is optional as there are no robust clinical trials evaluating this step, but it is frequently reapproximated for the theoretical prevention of bowel obstruction [19].

\section{Technique variations}

Sacrocolpopexies and SCerPs have many possible modifications. A variety of grafting materials have been used including nonabsorbable synthetic graft (eg, polypropylene, polyester, silicone rubber, polytetrafluoroethylene), absorbable synthetic graft (eg, polyglactin), and biologic (eg, autologous rectus fascia or fascia lata, cadaveric dura mater and fascia lata, xenoform porcine dermis) [20-23]. Grafts may be preformed or individually crafted, in 1 or 2 pieces ("Y" vs "L"-shaped grafts), fixed to the anterior sacrum with nonabsorbable and/or absorbable sutures or tacking/fixation devices, tunneled from the posterior broad ligament to the presacral space, or tensioned initially in a complete opening of the peritoneum on the right pelvic side wall from the presacral space to the posterior leaf of the broad ligament. The graft may also be a single piece that is sutured to the posterior vagina, rolled over and sutured to the vaginal apex or anterior vagina, and then secured to the sacrum. The graft may be attached to the anterior and/or the posterior fibromuscular layers of the vagina, attached to the vaginal apex (SCP) or anterior/posterior cervix (SCerP) only without more caudad extension, or both. Choices of suture for vaginal graft attachment include nonabsorbable, delayed absorbable, or barbed delayed absorbable. The number of sutures to the anterior sacrum (2-4), the vaginal apex or cervix (1 or 2), or the anterior or posterior vaginal wall (1 barbed suture with multiple attachment points or many interrupted sutures) can vary, as can the location of graft or graft attachment on the anterior (anywhere from the apex to the bladder neck) or posterior vaginal wall (anywhere from the apex to the perineal body). Finally, the graft can be attached to the anterior and posterior vaginal walls transvaginally and attached to the sacrum through an abdominal or laparoscopic approach [24].

Another important variation on SCP is the extension of the posterior vaginal graft attachment point down to the perineal body for the purpose of perineal support [25]. The term "sacral colpoperineopexy" has been used in the literature to describe this variation $[26,27]$, and we recommend that the variation that attaches the posterior vaginal graft to the perineal body be a specific subterm underneath the broader term of "sacrocolpopexy," as this variation still meets the requirements for the definition of SCP. We recommend against the less specific term "perineopexy" for this technique of sacral colpoperineopexy, as it is easily confused with other perineal support procedures with different methods. Future directions for SCP and other procedures discussed in this document can be found in Appendix 1.

\section{Special terminology considerations}

In a patient with a uterus in situ, any route of hysterectomy can be followed by SCP as described previously. If a supracervical hysterectomy is chosen, the graft is usually attached to the cervix as well as the anterior and posterior vagina, and this is termed a SCerP. Some sources term this combination of supracervical hysterectomy and SCerP appropriately [28-30], whereas some sources use the term SCP interchangeably to describe this sequence of event [31]. We recommend the former.

\section{Summary}

SCP and SCerPs are apical prolapse procedures where the vagina or cervix, respectively, is bridged to the anterior longitudinal ligament of the sacrum at the level of S1-S2 using a graft. Surgeons should be clear with patients that the use of this terminology indicates a procedure performed in the intra-abdominal cavity and using some type of graft, to distinguish this surgery from procedures accomplished entirely through the vagina that use graft.

\section{Uterosacral ligament suspension}

Uterosacral ligament suspension (USLS) is defined as suspension of the vaginal apex to the unilateral or bilateral uterosacral ligament(s) (USL[s]) using suture. USLS was first described as a transvaginal procedure and is most commonly performed through a vaginal approach [32, 33], but laparoscopic modifications are reported [34, 35] and any abdominal entry approach (laparoscopic, robotic, or open) is possible for USLS. Uterosacral ligament suspension often is performed at the time of a hysterectomy because the USLs can be easily identified, but this technique can be used in posthysterectomy vaginal prolapse repair as well.

The concept of using the USLs to support the vaginal apex was first described in 1927 [32]. In 1957, a technique attaching the distal USLs to the vaginal apex with purse-string sutures to close an "enterocele" was described [36], and modifications were made to this technique to minimize suture number and risk of ureteral injury [33]. Most modern publications on USLS reference reports in the 1970s theorizing that prolapse is related to "isolated breaks" in the USLs rather than overall attenuation [37]. In 2000, 2 studies separately reported on a USLS in which 1 to 3 sutures were 
placed through each USL and then attached to the ipsilateral fibromuscular layer of the anterior and posterior vaginal apex [38, 39]. Most USLS trials use a modification of this ipsilateral technique [40].

\section{Procedure description}

Equipment As mentioned above, USLS can be performed vaginally, laparoscopically, or in an open abdominal approach. Laparoscopic techniques use various methods of suspending the vaginal apex to the USLs without using transvaginal suturing $[41,42]$ but must include the steps below for the term to be termed a USLS. Cystoscopy for assessment of ureteral patency is considered clinically mandatory during USLS because of a high prevalence of ureteral injury [43], but use of the term USLS does not automatically imply that this vital step has been performed, so surgeons should clarify the performance of cystoscopy in communications regarding the surgery.

The transvaginal approach requires standard vaginal surgery equipment, such as long instruments and additional light sources, which can reach deep into the pelvis. Many surgeons describe using a combination of delayedabsorbable suture and nonabsorbable suture, whereas others use only delayed and/or rapidly absorbable sutures. In the laparoscopic approach, an instrument to manipulate the vaginal cuff, laparoscopic needle drivers, and possibly a knot pusher are needed.

\section{List of steps}

Step 1: The peritoneal cavity is entered either transvaginally or abdominally. This suspension is often performed at the time of vaginal hysterectomy, in which case the peritoneal entry at the vaginal apex is used. For USLS remote from hysterectomy using a vaginal approach, the peritoneum is carefully entered through the vaginal vault to avoid bladder or bowel injury.

Step 2: The USLs are identified. In the ipsilateral USLS technique (Fig. 3), sutures are placed in the USLs proximal to the ischial spine.

Step 3: Sutures are placed through the posteromedial aspect of bilateral USLs and subsequently through the anterior and posterior vaginal cuff, ideally incorporating the fibromuscular layer. For absorbable sutures, the vaginal epithelium is incorporated into the suture as well; the vaginal epithelium is avoided with nonabsorbable sutures. Some authors describe performing a separate closure of the posterior cul-de-sac during USLS [38, $44,45]$, but this step is not itself considered a procedure for prolapse correction or necessary for use of the term USLS.

\section{Technique variations}

In another common variation, the USLs are plicated across the midline using sutures (Fig. 4). Sutures are passed first through the epithelium and fibromuscular layers of the anterior or posterior vaginal cuff, through the ipsilateral USL, reefed across the posterior peritoneum to the contralateral USL, through the contralateral USL, and again through the vaginal fibromuscular layer and out through the vaginal epithelium. Although this technique was originally described to close the posterior cul-de-sac [36, 46], this midline plication technique is frequently used to address apical vaginal prolapse and is appropriately termed USLS if the USLs are incorporated proximal to the level of the ischial spines.

The ipsilateral USLS technique is typically performed with 1 to 3 sutures per side for a total of 2 to 6 sutures, whereas the midline plication technique uses 2 to 3 sutures across the midline with additional sutures if the peritoneum is being closed above the USL plication.

Laparoscopic or open approaches vary, but common features include identification of the USLs and mobilization of the ureters laterally and away from the site of planned USL suture placement. Sutures are then passed through the USLs and through the vaginal cuff incorporating the fibromuscular layers similar to the vaginal approach $[34,35]$. Another modification of the USLS is an extraperitoneal technique, although this is less common $[47,48]$. Unilateral USLS is also possible when sutures on one side of a planned bilateral suspension must be removed and replacement is avoided [49].

\section{Special terminology considerations}

Attachment of a graft from the vaginal apex to the presacral space, even in the proximity of where the USLs insert, would more appropriately be termed a SCP. Attaching the vaginal apex to the peritoneum only, without identification and confirmation of suture placement into the USLs, would not qualify as a USLS. In addition, reattachment of the vaginal apex or cuff to the cut ends of the USLs distal to the level of the ischial spines, as at the end of a hysterectomy, is not considered a USLS.

\section{Summary}

Uterosacral ligament suspension suspends the apex of the vagina to the USLs with the use of suture material. A wide variety of approaches to access the USLs and place the sutures exist, but the core principle is utilization of the USLs for apical support. 


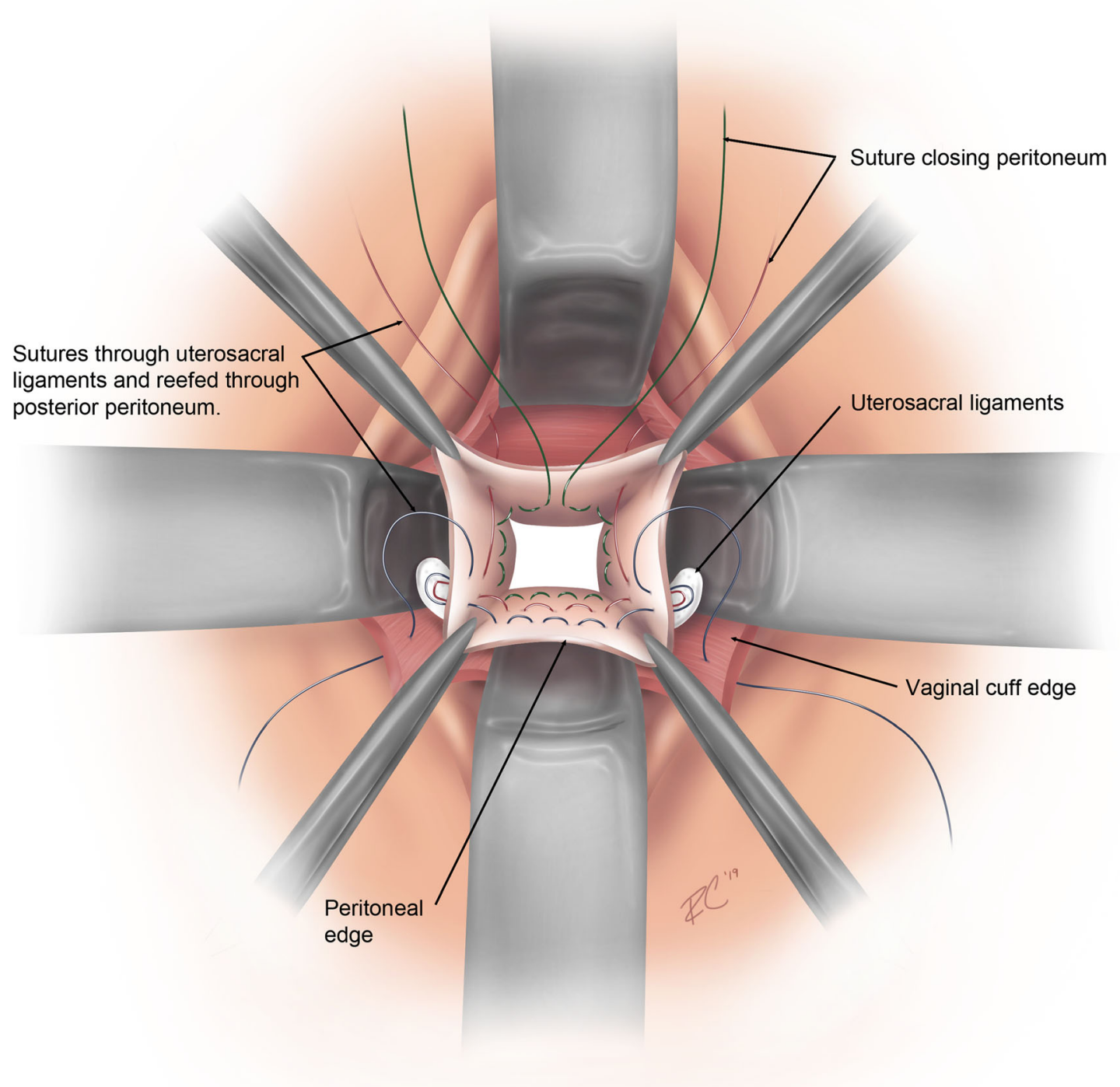

Fig. 3 Uterosacral ligament suspension using the ipsilateral technique variation

\section{Sacrospinous ligament fixation with or without graft}

Sacrospinous ligament fixation (SSLF) is defined as suspension of the vaginal apex to the unilateral or bilateral sacrospinous ligament(s) (SSL[s]) using suture (Fig. 5). Similarly, SSLF with graft is the suspension of the vaginal apex to the unilateral or bilateral SSL(s) with incorporation of graft (Fig. 6). Typically, the vaginal apex is attached to one or both $\operatorname{SSL}(\mathrm{s})$ at a location at least 2 to $3 \mathrm{~cm}$ medial to the ischial spine [50]. The SSLs are interconnected with the coccygeus muscles, so the anatomic term "sacrospinous ligament-coccygeus muscle complex" is used in many publications describing SSLF. Though initially described in 1958 [51], the procedure gained popularity in 1968 when further detailed in the literature [52].

\section{Procedure description}

Equipment Sacrospinous ligament fixation is most commonly performed transvaginally and can be performed without entering the peritoneal cavity (Table 2). Although SSLF is usually performed transvaginally, a laparoscopic approach has been described [53]. If a uterus is present, the SSLF can be performed concurrently with a hysterectomy or leaving the uterus in place (see "sacrospinous hysteropexy" hereinafter).

With direct visualization, ligament perforation and retrieval can be aided with specific devices, such as a Deschamps ligature carrier or Miya hook [54, 55]. In an attempt to decrease morbidity associated with the dissection required for direct visualization of the SSL, instruments have been used for that 
Cuff of vagina and tied down sutures plicating uterosacral ligaments.

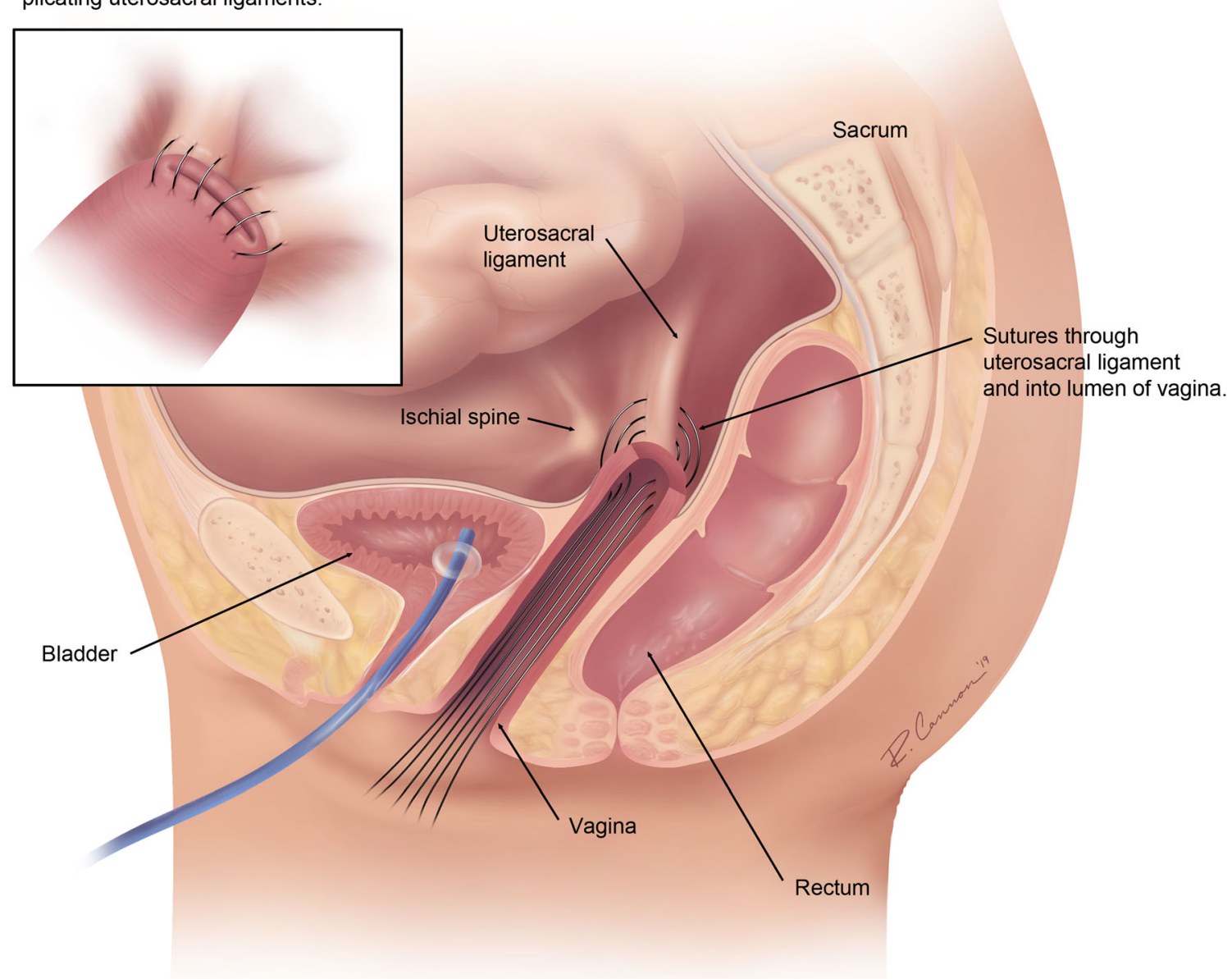

Fig. 4 Uterosacral ligament suspension using the midline plication technique variation

facilitate SSL suture placement by "palpation" alone, such as the i-stitch ${ }^{\circledR}$, Endo Stitch ${ }^{\circledR}$, and Capio ${ }^{\circledR}$ devices [56].

\section{List of steps}

Step 1: The SSLs are located in the pararectal space, contiguous with the retropubic space, and may be approached through an anterior, posterior, or apical vaginal incision. The posterior approach develops the rectovaginal space and the rectal pillars are perforated at the level of the ischial spine, thus gaining access to the pararectal space. The anterior approach develops the vesicovaginal space with entry of the retropubic space via perforating the arcus tendineus fasciae pelvis (ATFP), preferably near the pubic bone insertion. This is followed by development of the paravaginal space until the ischial spine is reached, leading to the identification of the SSL [57].

Step 2: The SSL is identified by visualization and/or palpation and dissected [54]. If the peritoneal cavity is entered, the SSL can be approached through the posterior visceral peritoneum. This peritoneum is "peeled off" at 7 o'clock (for access to right SSL) and a cephalad finger motion allows reaching the SSL [55].

Step 3: $\quad$ Sutures are passed through the SSL using either direct visualization or palpation $[58,59]$.

Step 4: These sutures are then passed through the fibromuscular layer of the vaginal apex with or without incorporating the vaginal epithelium. 
Cuff of vagina and tied down sutures

to the sacrospinous ligament.

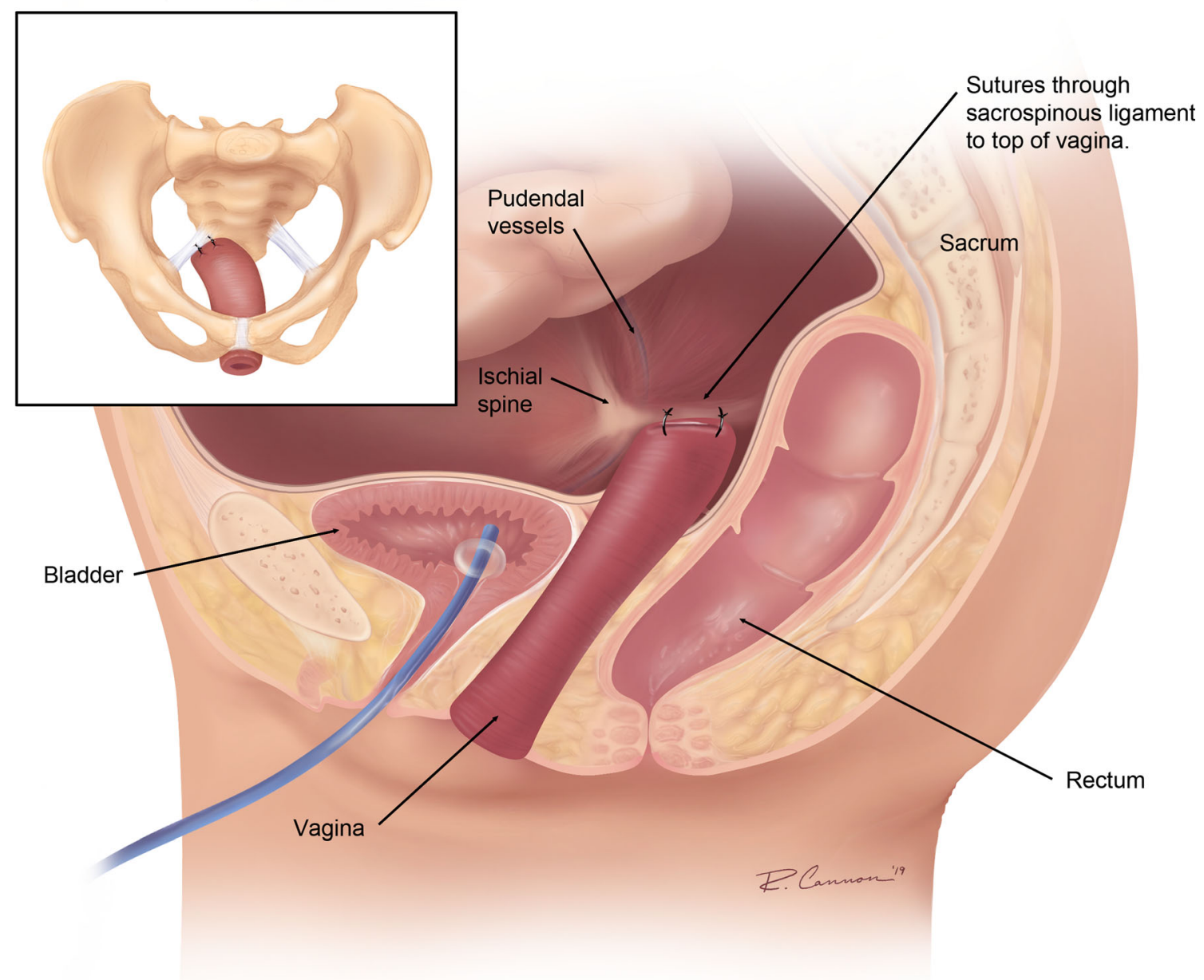

Fig. 5 Sacrospinous ligament fixation

The vaginal apex could be at the location of the former apical vaginal scar (posthysterectomy) or a new location on the cephalad vagina determined by the surgeon to be more ideal for restoring anatomy with this repair.

Step 5: Sutures are tied to suspend the vaginal apex with or without additional plication of the fibromuscular layers of the anterior and/or posterior vaginal walls.

\section{Technique variations}

If graft is being used in a SSLF, the sutures passing into the SSL(s) may be part of a prefabricated graft product wherein the sutures have bullets to aid in their passage through the SSL(s) [60-62]. Sutures placed in the SSL may also be passed through a graft without any preattached sutures [63]. One example of SSLF with graft in the past used an anterior vaginal approach and involved an arced strap of type 1 polypropylene mesh that was attached to the bilateral SSLs by nonabsorbable sutures with bullets and fixed in the midline to the cervical stroma or vaginal apex [64]. Grafts of this type were withdrawn from use in the US market in 2019 (see Appendix 2). Grafts used in SSLF may also be attached at other points in the pelvis or additional graft pieces may be used, but the term SSLF with graft is appropriate if the graft material is affixed to one or both $\operatorname{SSL}(\mathrm{s})$.

Although the fixation near the ischial spine has been used in SSLF [65], most studies recommend a point of attachment 2 to $3 \mathrm{~cm}$ medial to the ischial spine to avoid neurovascular structures $[55,60]$. Segments of the vagina 


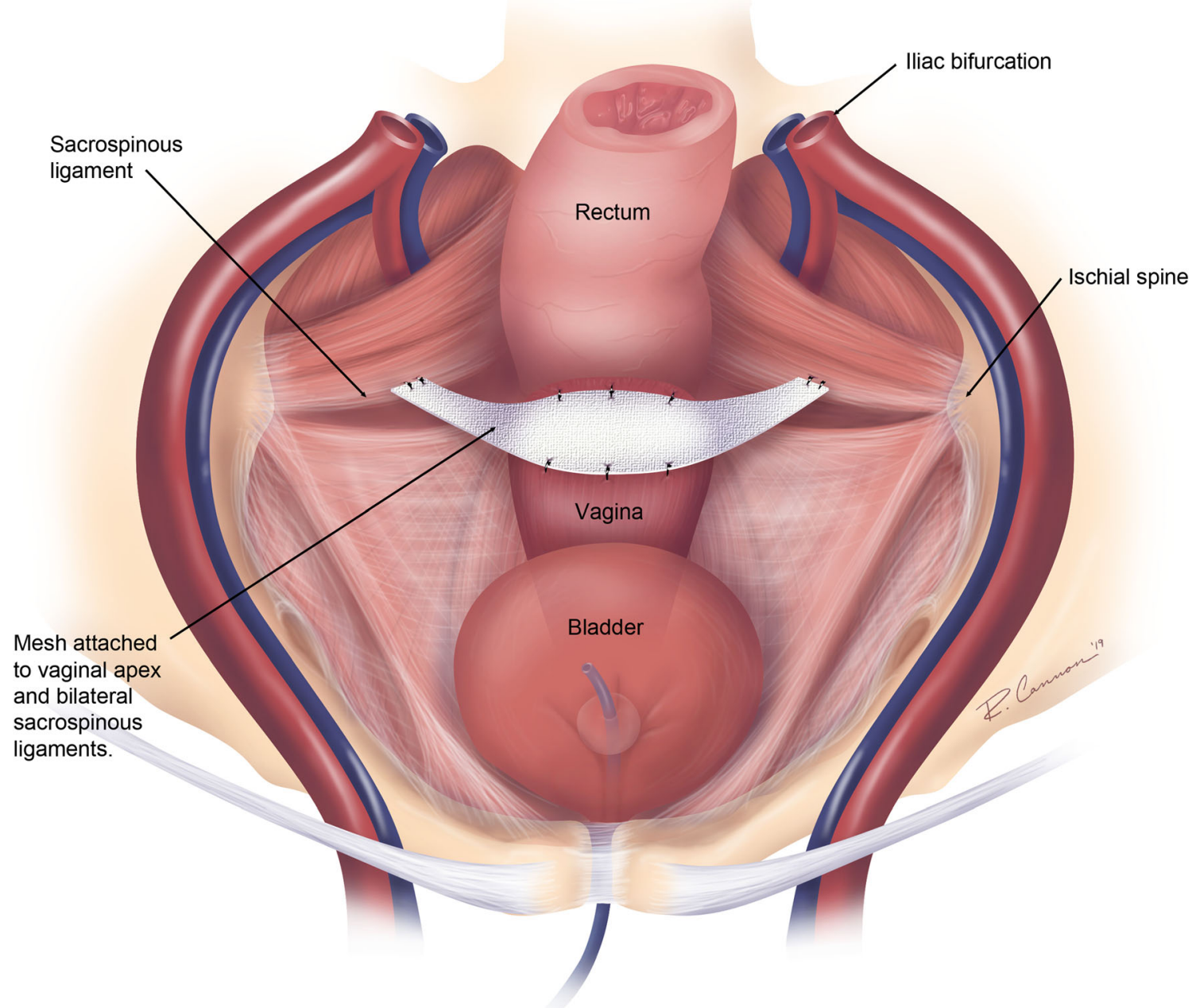

Fig. 6 Sacrospinous ligament fixation with graft using the technique variation with graft affixed to the anterior vaginal wall and the arms of the graft fixed to bilateral sacrospinous ligaments

incorporated into the suspension can be the posterior and/ or anterior vaginal walls. The vaginal epithelium may or may not be included in the SSL sutures. Part of the vaginal apex may be excised if there is redundant vaginal length [66].

Suture material used for the suspension can be nonabsorbable (when vaginal epithelium is not incorporated) and/or delayed absorbable (when the sutures are tied within the vaginal lumen). The number of sutures placed in the SSL ranges from 1 to 4 . Multiple sutures can incorporate anterior and posterior walls even in unilateral SSL [55]. Expert opinion suggests tying the knots in direct contact with the SSL.

Initial reports described bilateral suspension of the vaginal apex to both SSLs [52]. Some surgeons advocate unilateral suspension, usually right sided, as it theoretically decreases the incidence of rectal injury [54, 55, 67]. Left-sided SSLF has also been described in primary or repeat procedures [60].

\section{Special terminology considerations}

As noted previously, the distance of the placement of suture or graft from the ischial spine varies, but the ligament itself must be incorporated and confirmation of SSL placement must be a part of the procedure.

\section{Summary}

Sacrospinous ligament fixation is a procedure that attaches the vaginal apex to one or both SSL(s) either with suture material or with the incorporation of graft. This procedure can be performed without entry into the peritoneal cavity, typically through a transvaginal approach.

\section{Iliococcygeus fixation}

Iliococcygeus fixation (IF) is defined as a suspension of the vaginal apex to the unilateral or bilateral medial parietal fascia 
of the iliococcygeus muscle(s) medial to the ischial spine using suture material (Fig. 7). Fixation to the iliococcygeus fascia is typically caudad to the ischial spine to minimize the risk of neurovascular injury [68].

In 1963, suspension of the vaginal cuff to the iliococcygeus fascia was first suggested in situations when the USLs were attenuated and not adequate for support [69]. Another author then revised the procedure and reported experience using bilateral iliococcygeus fascia suspension for the vaginal vault [70]. This technique has been used primarily when the uterus is absent but may also be used concurrent with hysterectomy or theoretically with the uterus in place [71].

\section{Procedure description}

Equipment Iliococcygeus fixation is always approached transvaginally but does not require as extensive of a dissection of the pararectal spaces as does SSLF [54, 71-74] and does not use the intraperitoneal space as is most often the case in USLS. Therefore, vaginal equipment is needed (Table 2), but the depth of lighting and retraction needed may be less extensive in IF than in SSLF or USLS.

\section{List of steps}

Step 1: The pararectal space is approached through a vaginal incision. When the patient has had a prior hysterectomy, a midline vertical incision is made in the posterior vaginal wall. If a hysterectomy is being performed or the uterus is in place, a posterior vertical incision in the vaginal epithelium near the apex can be performed while still avoiding the vaginal cuff repair or cervix, respectively.

Step 2: The posterior vaginal epithelium is dissected from the underlying rectovaginal fibromuscular layer [71]. The pararectal spaces are entered and the ischial spines palpated bilaterally. The levator ani muscles and their parietal fascia are identified lateral to the rectum and anterior to the ischial spine.

Step 3: Several interrupted sutures are placed on the parietal fascia of the iliococcygeus on each side caudad to

Sutures attaching vaginal cuff

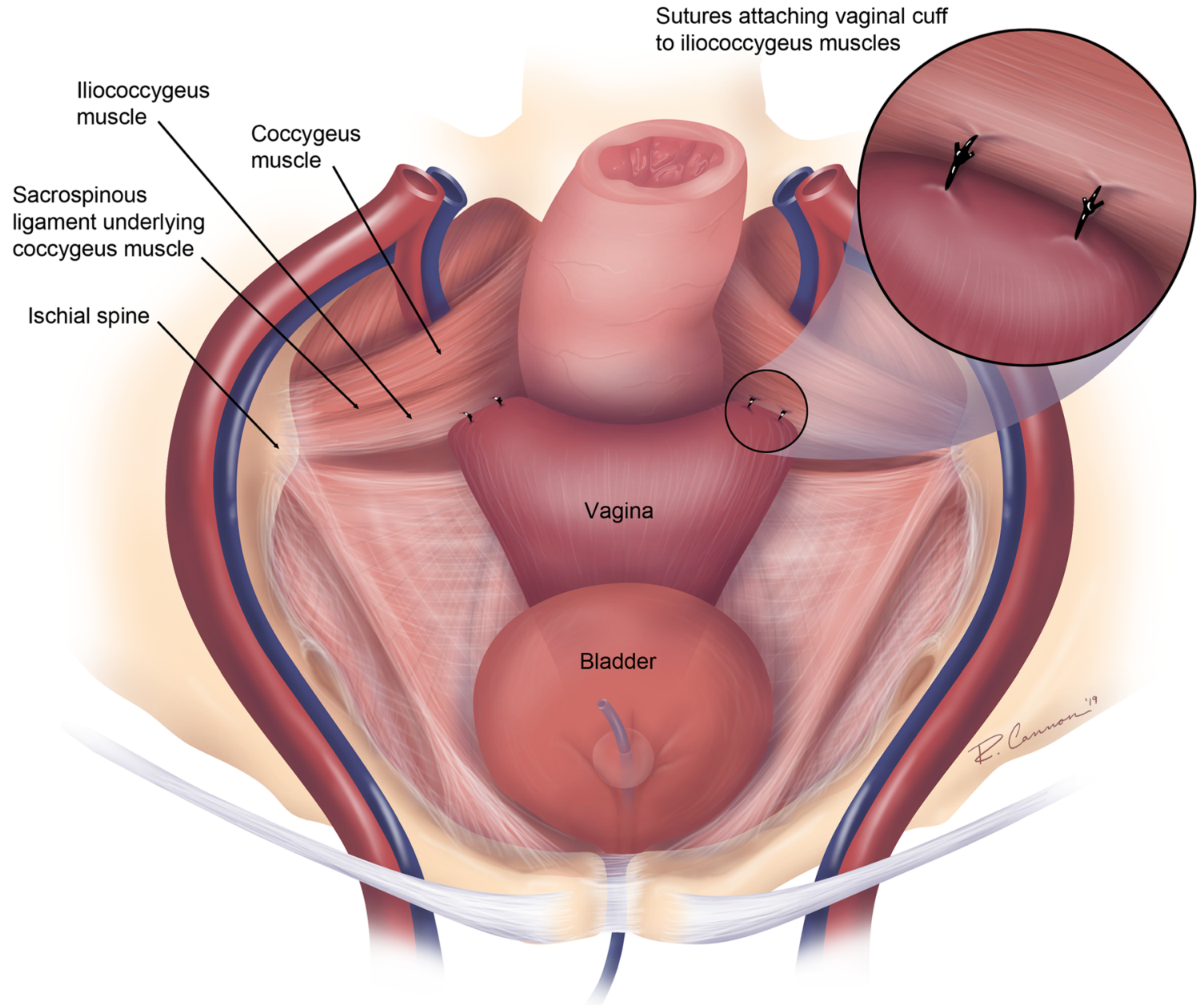

Fig. 7 Iliococcygeus fixation 
the ischial spines along an imaginary line connecting the ischial spine and the perineum.

Step 4: The vaginal walls are then attached bilaterally to the IF sutures. The highest IF suture is placed at the vaginal apex, and the others are passed to coincide with the lateral vaginal fornices, each one approximately $2 \mathrm{~cm}$ more caudad than the previous suture.

Step 5: The sutures are tied down to suspend the vaginal apex.

\section{Technique variations}

Iliococcygeus fixation can be unilateral or bilateral, and fixation sutures may be nonabsorbable or delayed absorbable. The iliococcygeus fascia $1 \mathrm{~cm}$ inferior to the ischial spine is the site of choice, but any location in the iliococcygeus fascia or both fascia and muscle can also be used.

\section{Special terminology considerations}

As the iliococcygeus muscle thickness is thin $(2.54 \mathrm{~mm})$ in anatomic studies [75], it is impossible for surgeons to distinguish if only the parietal fascia or both the fascia and muscle are in the suture, so both situations are appropriately termed an IF.

\section{Summary}

Iliococcygeus fixation suspends the vaginal apex to the parietal fascia of the iliococcygeus muscle through a transvaginal approach. Procedures that suspend from other ligaments, even ligaments in close approximation to the iliococcygeus muscle, such as the SSL or the ATFP, would not qualify for the use of the term IF.

\section{Hysteropexies}

Anatomical studies have established that the uterus is not a cause of prolapse [76], and it is known that hysterectomy is not itself a prolapse correction surgery [77]. Hysteropexies are prolapse procedures that suspend the uterine cervix or isthmus without removal of the uterus [78].

Several types of hysteropexies are performed worldwide. Sacrohysteropexy (SHP) refers to suspension of the uterine cervix or isthmus to the anterior longitudinal ligament of the sacrum using a graft, with possible incorporation of the graft into the anterior and/or posterior vaginal walls, with preservation of the uterine body $[79,80]$. Anterior abdominal wall hysteropexy (AAWHP) is defined as suspension of the uterine cervix or isthmus and possibly the fibromuscular layer of the anterior vaginal wall to the anterior abdominal wall, with or without utilization of a graft, with preservation of the uterine body [81-84]. Transvaginal hysteropexies include uterosacral hysteropexy (USHP), sacrospinous hysteropexy (SSHP), and the Manchester procedure. Uterosacral hysteropexy is defined as suspension of the uterine cervix or isthmus to the unilateral or bilateral USL(s) using suture with preservation of the uterine body [85-89]. Sacrospinous hysteropexy is defined as suspension of the uterine cervix or isthmus to the unilateral or bilateral SSL(s) using suture with preservation of the uterine body [90, 91], whereas SSHP with graft is the same with incorporation of graft [62, 92-94]. The Manchester procedure is defined as the shortening or the amputation of the uterine cervix with preservation of the uterine body and plication of the USLs extraperitoneally caudad to this amputation [95-98].

The earliest uterine preservation procedure described for prolapse was the obliterative LeFort colpocleisis in the early 1800s [99]. The first reconstructive procedure preserving the uterus was the Manchester procedure, first described in 1888 by authors in Manchester, England and subsequently documented in a case series from 1936 to 1955 in Iowa State Hospitals [96]. Historically, hysteropexies have involved the same principles as other prolapse procedures that suspend the vagina (colpopexies), including use of the same anatomical attachment structures, such as the SSLs or USLs. Thus, most colpopexy procedures could be modified to preserve the uterus [100]. For example, some of the earliest descriptions of SCP during the late 1950s in France do not recommend a hysterectomy for the vaginal suspension [101, 102]. Like other POP surgeries, hysteropexy has shifted toward less invasive approaches in recent years [103, 104], with vaginal hysteropexies using graft having the most publications in the literature [78].

\section{Procedure description by type of hysteropexy}

\section{Sacrohysteropexy}

Equipment Sacrohysteropexy may also be performed through open abdominal [86, 105], laparoscopic, or robotic approaches [93, 106, 107], so equipment will match the approach (Table 2).

\section{List of steps}

Step 1: The abdominal cavity is entered.

Step 2: The anterior and posterior cervix and vaginal walls are dissected.

Step 3: The presacral space is dissected to reveal the anterior longitudinal ligament.

Step 4: The graft is attached to the uterine cervix and/or isthmus and possibly also to the fibromuscular layer(s) of the anterior and/or posterior vaginal walls (Fig. 8). The anterior graft is passed unilaterally or bilaterally through the mesometrium of the 


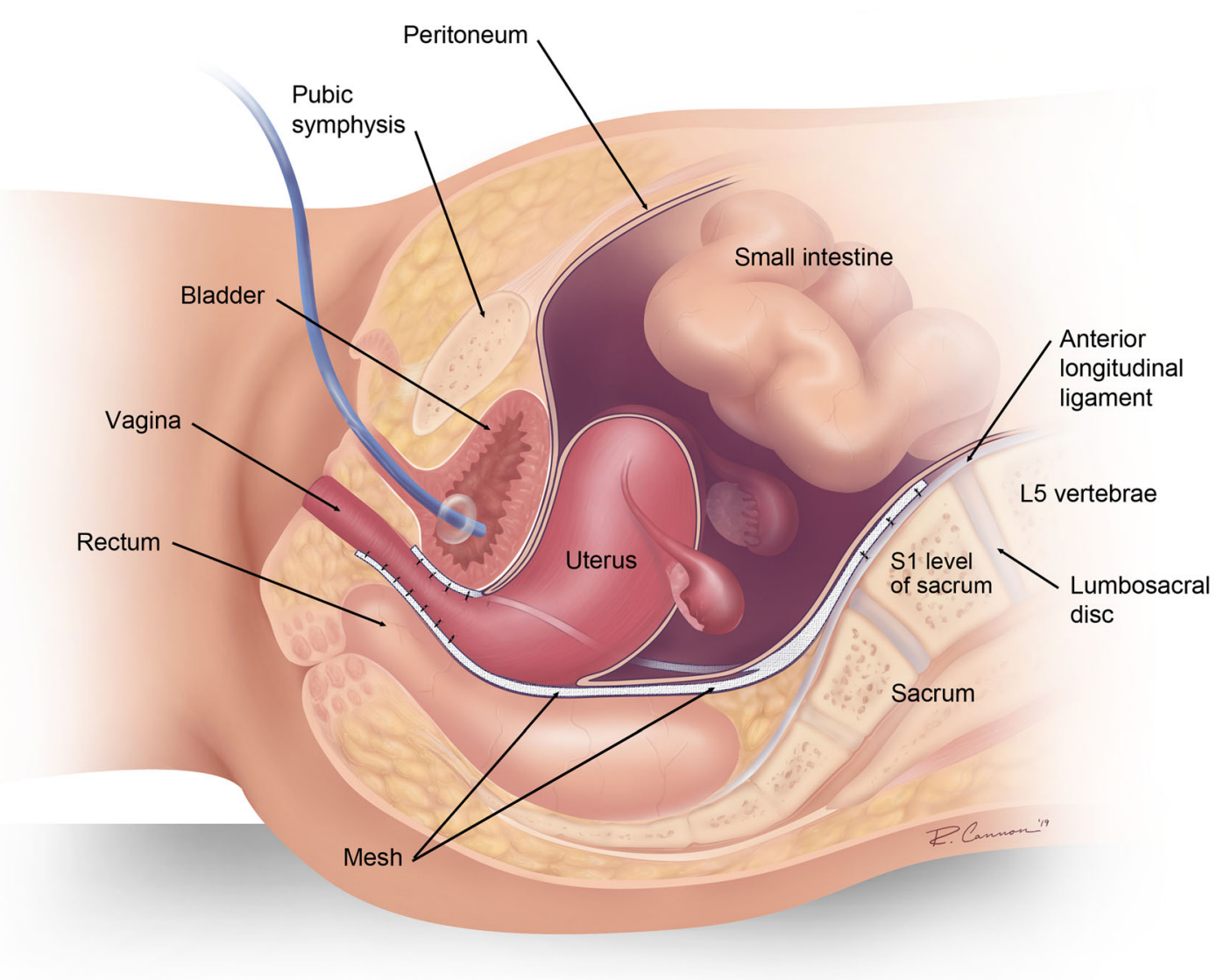

Fig. 8 Sacrohysteropexy

broad ligament. Placement of the graft only posteriorly has also been described.

Step 5: The base of the graft(s) opposite the end(s) attached to the vagina is/are attached to the anterior longitudinal ligament of the sacrum at the S1 level.

Step 6: Closure of the peritoneum over the graft is optional as in SCP and SCerP.

Technique variations Variations mostly are similar to those in SCP and SCerP, with additional variations on technique of graft passage through the broad ligament as discussed previously.

\section{Anterior abdominal wall hysteropexy}

Equipment These procedures are described with or without graft, and approaches are open abdominal (with or without entry into the intraperitoneal space depending on whether the structure for suspension is retroperitoneal), laparoscopic, or robotic. Equipment is particular to the approach, fixation points, and material used for fixation.

\section{List of steps}

Step 1: Access to the abdominal cavity abdominally or laparoscopically.

Step 2: The uterine cervix or isthmus, with or without involvement of the fibromuscular layers of the anterior and/or posterior vaginal wall, is attached to various structures involving the anterolateral abdominal wall (Fig. 9). These anterolateral abdominal wall structures could include the round ligament, pectineal ligament, or a point near to the anterior superior iliac spine (ASIS).

Step 3: The retroperitoneal or retropubic space is closed (if relevant) and abdominal incisions are closed to exit the abdominal space.

Technique variations Variations on AAWHP include bilateral or unilateral suspension, suture and/or graft use, fixation of 


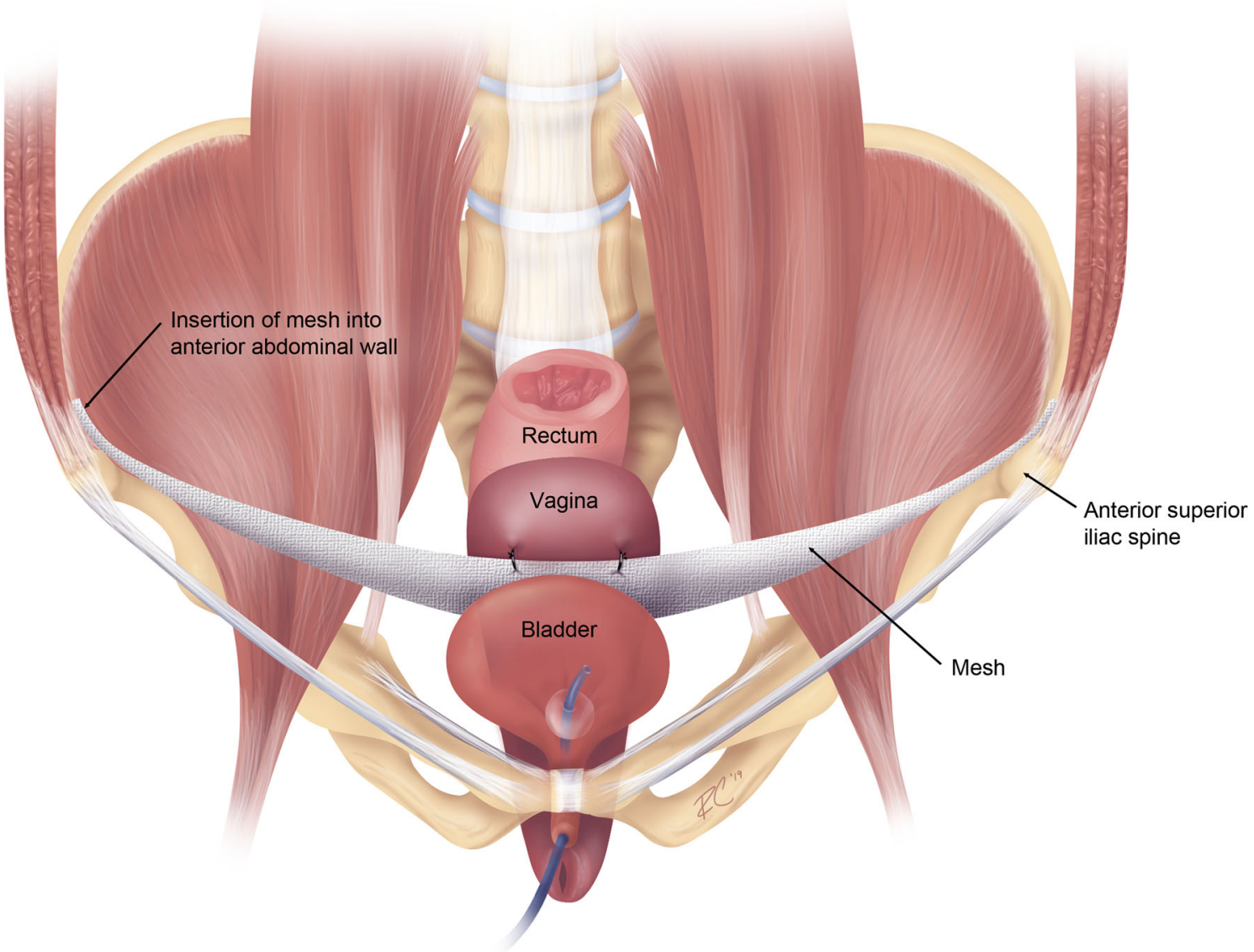

Fig. 9 Anterior abdominal wall hysteropexy using the technique variation fixing a graft to the anterior uterine isthmus with the lateral ends of the graft fixed to the anterior abdominal wall near the ASIS

graft (if used) to the uterine cervix or isthmus with sutures or adhesive, fixation of graft or sutures to the fibromuscular layers of the anterior and/or posterior vaginal wall, and the type of graft (autologous, biologic, or synthetic) or suture (nonabsorbable, absorbable, or both) used.

If a graft is being used for fixation to the anterior abdominal wall near the ASIS, this involves a synthetic graft fixed to the anterior cervix and the fibromuscular layer of the anterior vaginal wall after dissection of the vesicovaginal space. An instrument is inserted through an incision $4 \mathrm{~cm}$ posterior to the iliac spine and $2 \mathrm{~cm}$ above the iliac crest to retract the graft arms bilaterally in the retroperitoneal space caudad to the round ligaments. The graft is then symmetrically suspended to the anterior abdominal wall posterior to the ASIS [81, 108]. Closure of the peritoneum over the graft is optional. Laparoscopic approaches to this surgery have been described in the literature [81, 108] as well as an open approach [109].

For round ligament hysteropexy, critical steps involve entry into the peritoneal cavity and passage of sutures or graft through the round ligament at its uterine insertion point. The suture or graft is then extraperitonealized and passed out through the anterior abdominal wall fascia and fixed on the anterior abdominal wall fascia [82]. This procedure can also be performed using an autologous rectus fascial grafts on each side whose lateral ends are left fixed to the rectus fascia and whose medial ends are passed into the abdominal cavity through the deep inguinal ring for attachment to the posterior uterus just cephalad to the insertion of the USLs [84]. Synthetic grafts have been used for round ligament suspension as well [110]. Round ligament hysteropexy can be unilateral or bilateral suspension or plication of the round ligaments.

In a third form of AAWHP, pectineal ligament suspension, entry is made into the retropubic space to isolate the pectineal ligament, a nonabsorbable tape is fixed to the anterior uterine cervix, the ends of the tape are passed through the pectineal ligament on either side, and the ends of the tape are elevated to elevate the uterus per the discretion of the surgeon [83]. This surgery is described as being performed through open abdominal or laparoscopic approaches [83]. 


\section{Uterosacral hysteropexy}

Equipment Approaches to the USHP include open abdominal [86], laparoscopic [87-89], and transvaginal routes [104] with appropriate equipment for the approach (Table 2).

\section{List of steps}

Step 1: The intraperitoneal cavity is entered and visualization of posterior cul-de-sac is obtained.

Step 2: The USLs are identified and marked.

Step 3: The uterine cervix or isthmus (and possibly the apical vaginal apex) is attached to the USLs similar to USLS.

Step 4: The vaginal epithelium at the apex is closed.

Technique variations Possible variations to USHP are also those variations common in USLS and additionally include possible cervical amputation or trachelectomy.

\section{Sacrospinous hysteropexy}

Equipment This procedure is always performed transvaginally, with appropriate equipment this narrow space (Table 2).

\section{List of steps}

Step 1: The vaginal wall is entered anteriorly, posteriorly, or through the vaginal apex.

Step 2: Extraperitoneal dissection is performed to enter into the retropubic/pararectal space.

Step 3: One or both SSL(s) is/are palpated and/or visualized and dissected for access.

Step 4: The SSL(s) are attached to the uterine cervix or isthmus with sutures (Fig. 10) [111, 112].

Step 5: The vaginal epithelium is closed over the incisions.

Technique variations Variations on SSHP are similar to SSLF, including location of sutures on the SSL with the ideal

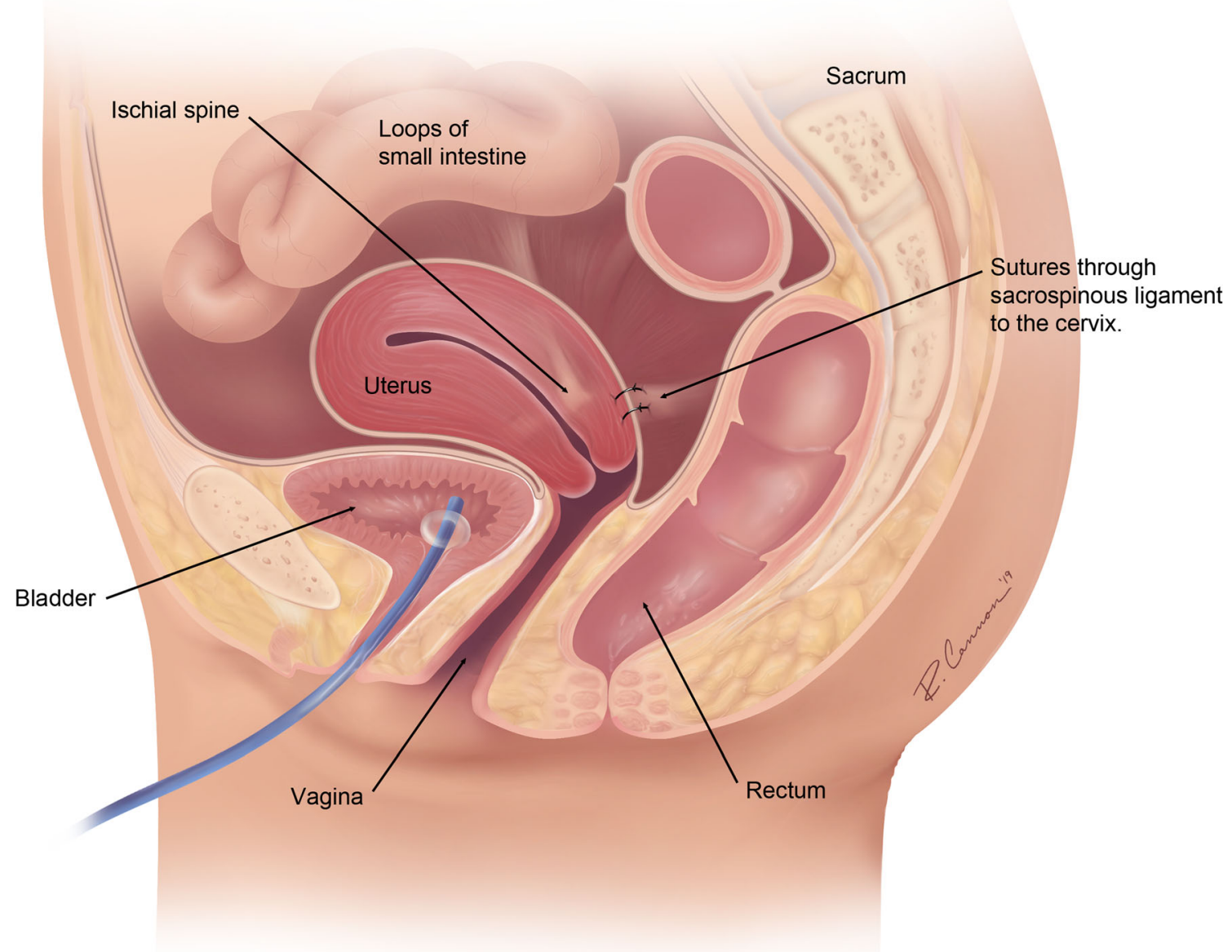

Fig. 10 Sacrospinous hysteropexy 
location at least $2 \mathrm{~cm}$ medial to the ischial spine [113]. There is also variation on the use of cervical amputation or trachelectomy. Sacrospinous hysteropexy can also be performed with graft materials, and the steps are similar to SSLF with graft. However, the graft is affixed to the cervix (and possibly to the vaginal apex) as well as to the SSL(s).

\section{Manchester procedure}

Equipment This procedure is always performed with a vaginal approach, and equipment for visualization and access of the vaginal apex are needed (Table 2).

\section{List of steps}

Step 1: Transvaginal trachelectomy is performed, with or without prior dissection of the vaginal epithelium and neighboring organs cephalad away from the cervix.

Step 2: The extraperitoneal insertion of the USLs into the cervix and apical vagina is visualized and/or palpated for identification of these ligaments.

Step 3: The USLs are plicated across the midline caudad to the level of the internal cervical os.

Step 4: The vaginal epithelium is closed over the apical incision.

Technique variations Variations of the Manchester procedure involve the number of sutures used to plicate the USLs across the midline, the use of nonabsorbable and/or absorbable sutures, and the extent of tissue removal with trachelectomy (total vs partial trachelectomy or number of centimeters of cervical tissue removed distally). Surgeons also vary in their method of trachelectomy, and use of cautery devices, such as electrode loops, has been described.

\section{Special terminology considerations}

Uterine preservation should not change the terminology stem of the procedure name, but the name would change to "hysteropexy" as opposed to "colpopexy" to indicate uterine preservation. Nearly all hysteropexies could have a corresponding colpopexy with similar steps, with modifications only to accommodate presence of a uterus. For example, procedures that typically use graft material for colpopexy, such as SCP, also use graft with uterine preservation.

\section{Summary}

Hysteropexies are prolapse repair procedures in which the uterine body is preserved, and the goal is to suspend the uterine cervix or isthmus. Hysteropexy surgical terms depend on the structures to which the uterus is being suspended.

\section{Anterior vaginal wall prolapse repairs}

The following 3 distinct surgeries are considered procedures specifically for anterior vaginal wall prolapse: anterior vaginal repair, anterior vaginal repair with graft (autologous graft, xenograft, or synthetic graft), and paravaginal repair. Although other previously discussed apical repair procedures could also be considered to address anterior vaginal wall prolapse, these surgeries specifically address the anterior vaginal wall.

\section{Anterior vaginal wall repair with or without graft}

Anterior vaginal repair is defined as repair of the fibromuscular layer of the anterior vaginal wall (Fig. 11), most commonly through either the subtechnique termed anterior colporrhaphy, defined as midline plication of the fibromuscular layer of the anterior vaginal wall, or the subtechnique of site-specific anterior vaginal repair, defined as the site-specific repair of specific defects in the fibromuscular layer of the anterior vaginal wall. Despite extensive variety in the literature and in practice [114], the core of what constitutes an anterior vaginal repair is the midline suture repair of the fibromuscular layer by one of these 2 techniques. In other aspects of the surgery, there is significant practice variation $[115,116]$.

In 1886, the first anterior vaginal repair with full thickness excision of vaginal epithelium and resuturing was described. Soon after in 1901, 2 additional authors described the support of the pelvic floor with prominence given to repair of the anterior vaginal wall [114]. Sitespecific anterior vaginal repair was a variation advocated in the 1990s, which was limited to reapproximation of breaks in the fibromuscular layer [117].

Anterior vaginal repair with graft is defined as the reinforcement of the fibromuscular layer of the anterior vaginal wall with implanted graft material. One of the first graftaugmented anterior vaginal repairs was performed with tantalum mesh in 1955, followed by use of collagen mesh in 1970 . In 1992, autologous tissue was used for correction of recurrent prolapse. In the late 1990s and early 2000s, there were reports released on the use of synthetic mesh in anterior vaginal wall prolapse repair preceding the US Food and Drug Administration (FDA) the FDA reports of 2008 and 2011 and subsequent concerns about the safety of these procedures $[118,119]$.

\section{Procedure description}

Equipment Anterior vaginal repair is always approached vaginally with appropriate equipment for vaginal visualization and retraction (Table 2). 
Fig. 11 Anterior vaginal repair

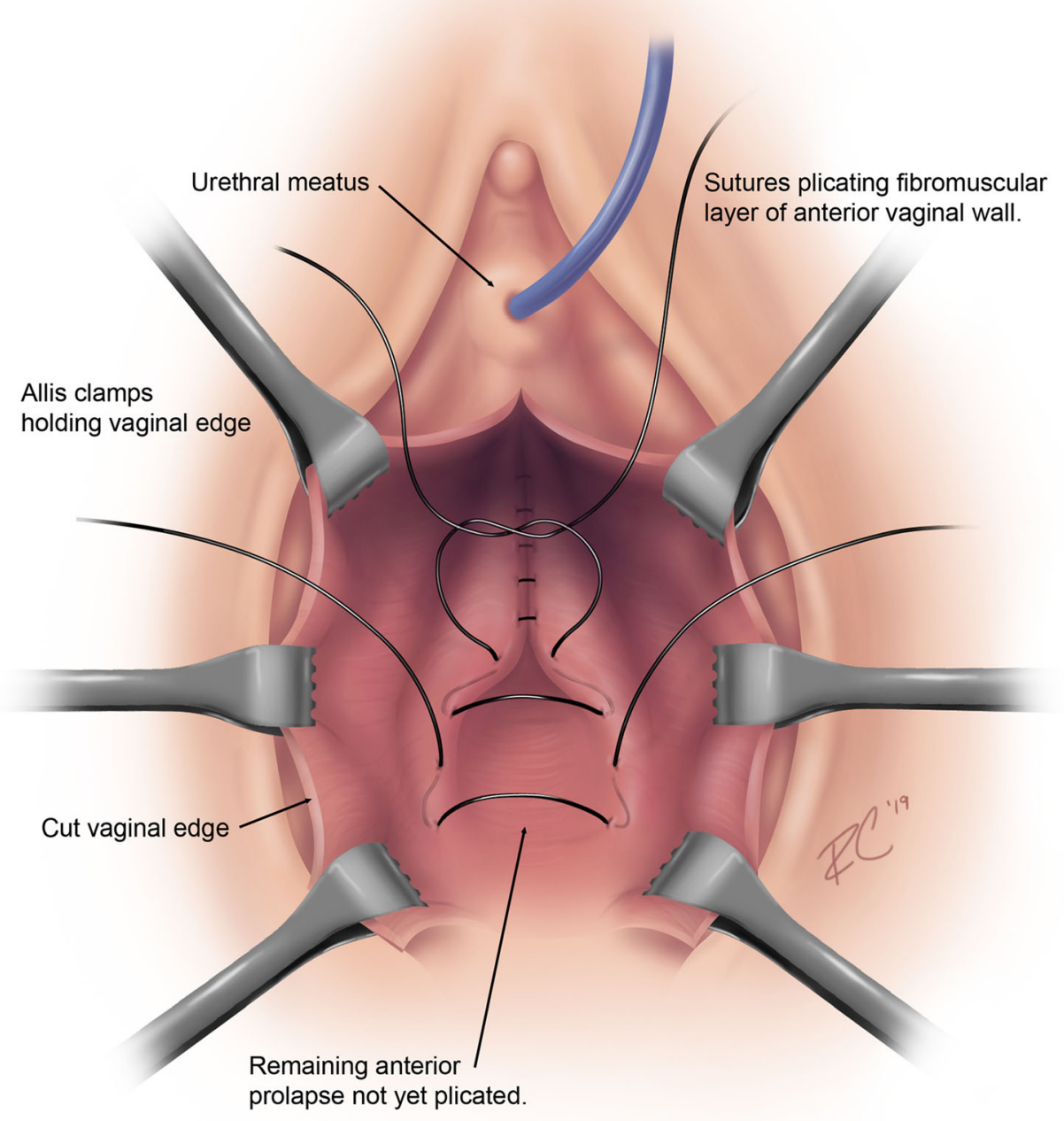

\section{List of steps}

Step 1: A longitudinal midline incision is made from the approximate location of the urethrovesical junction (typically $1-3 \mathrm{~cm}$ from the urethral meatus and often identified with use of an inflated bulb on a transurethral catheter) to the most proximal extent of the anterior vaginal wall prolapse. Often, the anterior vaginal wall is infiltrated with local anesthetic and/or a vasoconstricting agent for hydrodissection before the incision [120].

Step 2: The vaginal epithelium is mobilized from the underlying fibromuscular layer of the anterior vaginal wall, ideally continuing laterally to the ATFP or the inferior pubic rami [114]. The site-specific defect(s) in the fibromuscular layer of the anterior vaginal wall, if relevant, is identified.

Step 3: A series of sutures are placed to plicate the fibromuscular layer in the midline and/or suture is used to repair site-specific defects in this layer, reducing the anterior vaginal wall prolapse [121]. One or more plicating layers can be placed.

Step 4: The vaginal epithelium is closed with or without trimming the edges.

Technique variations If a graft is being used in an anterior vaginal repair, deeper injection of a hydrodissecting agent may help aid in the full-thickness fibromuscular layer dissection 
often recommended with graft placement in this space. The fibromuscular layer of the anterior vaginal wall may or may not be plicated at the time of anterior vaginal repair with or without graft placement, but plication of the fibromuscular layer is necessary to use the subterm "anterior colporrhaphy." Graft fixation may be determined by the graft type or may be individually crafted to the patient's anatomy. Tensor fascia lata or rectus fascia may be used as autografts. Allografts can be harvested from human cadavers (fascia lata and acellular dermal matrix), and xenografts of bovine or porcine acellular extracts are harvested from pericardium, dermis, and intestine submucosa and contain collagen and extracellular matrix. Attachment points can be to the anterior vaginal fibromuscular layer only ("graft patch") or to the ATFP, the obturator membrane and/or obturator muscles, the iliococcygeus muscle(s), the $\operatorname{SSL}(\mathrm{s})$, or some combination of the above. Attachment can be accomplished with absorbable or nonabsorbable sutures or with bullets on a prefabricated anterior synthetic mesh product.

The incisional type and extent can vary considerably in anterior vaginal repair without changing the terminology. Some surgeons opt for an elliptical incision, excising some vaginal epithelium at the start of the procedure. When anterior vaginal repair follows vaginal hysterectomy, some surgeons perform a triangular incision extending from the urethrovesical junction to the vaginal apical incision with the base of the triangle at the proximal portion of the anterior vaginal wall. Dissection of the vaginal epithelium from the underlying tissue can be conducted with sharp, blunt or mixed technique, and the plane of dissection may be superficial, dissecting the vaginal epithelium off all underlying tissue; deep, dissecting all tissue off the bladder; or both, wherein a free layer of fibromuscular tissue is dissected off both vagina and bladder. Plication of the fibromuscular layer (anterior colporrhaphy), if being performed, can be conducted with absorbable or delayed absorbable sutures, and can be interrupted, continuous, or in a purse string, followed by closure of the vaginal epithelium [122].

\section{Special terminology considerations}

In the case of anterior vaginal repair with graft, communications with the patient and in the medical record should distinguish what type of graft was used and what attachment points were used, thus distinguishing clearly from an anterior vaginal repair without graft and communicating the relevant anatomy.

\section{Summary}

Anterior vaginal repair is a specific repair of the anterior vaginal wall fibromuscular layer, most commonly with a midline plication technique, which defines the subterm anterior colporrhaphy. Anterior vaginal repair with graft refers to graft implanted in the vesicovaginal space to improve mechanical strength of an anterior repair while preserving the basic surgical approach.

\section{Paravaginal repair}

Paravaginal repair is defined as the approximation of the lateral fibromuscular layer of the anterior vaginal wall to the tendinous arch of the pelvic fascia (ATFP) (Fig. 12). In 1909, a transvaginal procedure consistent with a paravaginal repair was reported that involved anterior vaginal wall plication sutures reaching to the ATFP as opposed to merely plicating the fibromuscular layer as is performed in anterior colporrhaphy [123]. The same procedure was also suggested in 1976 when it was noted that detachment of the pubocervical fascia from the ATFP is associated with descent of the lateral part of the anterior wall. In 1981, the same author reported the outcome of paravaginal defect repair, and over a decade later, a case series reported the results of surgical repair of bilateral paravaginal defects $[37,117,124]$.

\section{Procedure description}

Equipment This procedure can be accomplished through an open abdominal, laparoscopic, or a vaginal approach. Regardless of approach, surgery always takes place in the retropubic space and equipment should be appropriate to visualize, enter, and retract within this space (Table 2).

\section{List of steps}

Step 1: In the abdominal or laparoscopic approach, the patient should be positioned in low dorsal lithotomy position to allow for a combined abdominal and vaginal approach. In the vaginal approach, standard lithotomy is most appropriate.

Step 2: A transurethral catheter is inserted into the bladder.

Step 3: The retropubic space is accessed via the peritoneal cavity through laparoscopic incisions, via the anterior abdominal wall by entering the rectus fascia through an open abdominal incision, or via perforation of the ATFP through a vaginal incision.

Step 4: After opening the retropubic space, the bladder and vagina are retracted medially for better visualization of the lateral retropubic space. Gentle blunt dissection is used to visualize the ischial spine and ATFP.

Step 5: Either delayed absorbable or nonabsorbable sutures are placed from the fibromuscular layer of the anterior vaginal wall to the ATFP. These sutures are placed bilaterally and extend from the ischial spine to the pubic bone insertion of the ATFP. Although the number of sutures varies (3-5 per side), they are typically placed at $1-\mathrm{cm}$ intervals. If placing nonabsorbable sutures from the abdominal side 


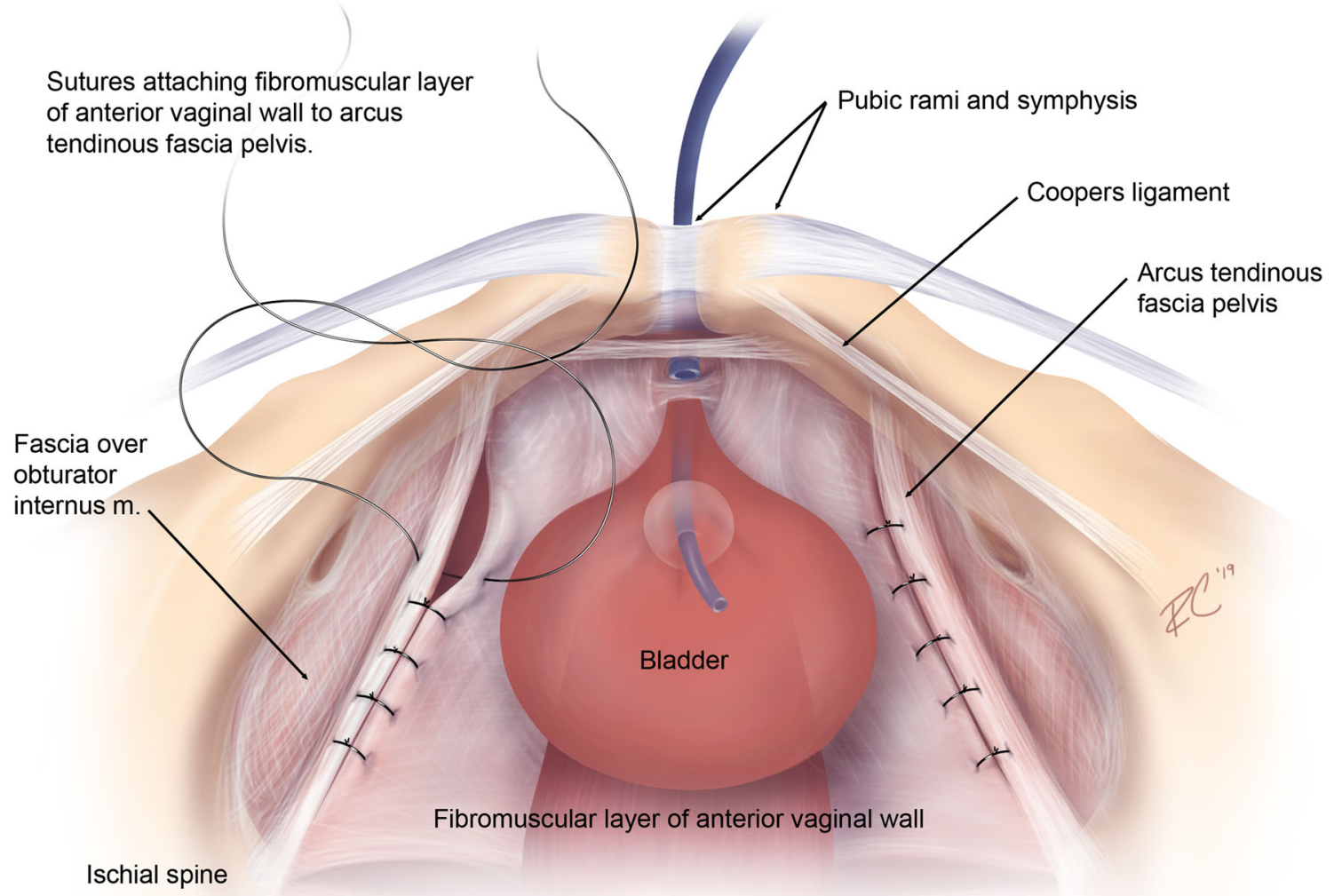

Fig. 12 Paravaginal repair

(abdominal or laparoscopic approach), care is taken to avoid placing suture into the vaginal lumen. In the abdominal or laparoscopic approach, suture placement is facilitated by insertion of 2 fingers of the surgeon's nondominant hand into the vagina and retraction of the bladder with an atraumatic instrument.

Step 6: The sutures are sequentially tied [121, 125].

Step 7: The retropubic space is closed either by reperitonealizing the bladder (laparoscopic approach), closing the rectus fascia (open abdominal approach), or closing the vaginal epithelium (vaginal approach).

Step 8: In the abdominal or laparoscopic approaches, subcutaneous and/or skin closure is performed.

Technique variations In the vaginal approach, the surgeon may or may not incorporate the vaginal epithelium in the sutures attaching the fibromuscular layer of the anterior vaginal wall to the ATFP. If the epithelium is being incorporated, suture placement uses a 3-point closure that places the sutures in the ATFP, followed by suture passage through the fibromuscular layer and finally through the vaginal epithelium. A midline vaginal fibromuscular layer plication (anterior vaginal repair with the anterior colporrhaphy technique) may or may not be performed concurrently. The direction of suture placement may vary by approach; in the vaginal approach, the surgeon usually starts placing sutures as close to the urethral meatus as possible and works posteriorly toward the ischial spine. In the abdominal or laparoscopic approach, either direction may be used.

\section{Special terminology considerations}

Because of the retropubic location of this procedure and its specific support of the anterior vaginal wall, this procedure is often misconstrued as a surgery for stress urinary incontinence. To avoid terminology confusion, care should be taken to link paravaginal repair to the diagnosis of anterior vaginal wall prolapse and avoid terms such as "bladder lift" or "sling" in discussion of this procedure.

\section{Summary}

Paravaginal repair is the reattachment of the fibromuscular layer of the lateral anterior vaginal wall to the ATFP and 
may be achieved with vaginal, laparoscopic, or abdominal approach.

\section{Posterior vaginal wall prolapse repairs}

Anatomical structures involved in support of the posterior vaginal wall are the peritoneum of the cul-de-sac, the rectum, and the perineum [126]. Thus, the loss of fibromuscular support overlying the rectum, laxity and separation of the levator ani plate with widening of the levator hiatus, and tearing or separation of the perineal musculature are deficiencies associated with posterior vaginal wall prolapse. Surgeries to correct these deficits involve the following 4 distinct terms: $(a)$ posterior vaginal repair [127], $(b)$ posterior vaginal repair with graft [128], (c) perineal repair, and $(d)$ levator plication.

\section{Posterior vaginal repair with or without graft}

Posterior vaginal repair is defined as repair of the fibromuscular layer of the posterior vaginal wall (Table 1, Fig. 13), either through a technique of midline plication of the vaginal fibromuscular layer, which defines the subterm posterior colporrhaphy or through a technique of deification and site-specific repairs of defect(s) in the fibromuscular layer defining the subterm site-specific posterior vaginal repair. The term posterior colporrhaphy was originated in Heidelberg, Germany, in 1867, when a surgeon applied it to 30 cases of uterine prolapse [129]. However, this term is now used to describe surgeries to correct posterior vaginal wall prolapse that plicate the fibromuscular layer in the midline. Site-specific posterior vaginal repair was a variation advocated in the 1990s, which was limited to reapproximation of breaks in the fibromuscular layer [130]. Plication of the levator ani muscle in the midline was formerly considered part of the posterior vaginal repair; it is now considered a separate procedure [127].

Posterior vaginal repair with graft is defined as reinforcement of the fibromuscular layer of the posterior vaginal wall with implanted graft material (Table 1). The graft material is meant to act as a scaffold and potentially replace or augment the fibromuscular layer of the posterior vaginal wall [128].

\section{Procedure description}

Equipment Posterior vaginal repair is typically performed transvaginally, but transabdominal (open or laparoscopic) [131], transanal, and transperineal [132, 133] approaches are also described. Equipment must be appropriate for the spaces entered (Table 2). All published studies on posterior vaginal repair with graft have used a transvaginal approach.

\section{List of steps}

Step 1: A longitudinal incision is made in the posterior vaginal epithelium. The posterior vaginal wall is often infiltrated with local anesthetic and/or a vasoconstricting agent for hydrodissection before the incision.

Step 2: The vaginal epithelium is dissected to expose the underlying fibromuscular layer, and this dissection is carried out laterally to the medial aspect of the levator ani muscles and inferiorly to the perineal body. If a graft is going to be used, an additional dissection may be performed opening the space between the fibromuscular layer and the rectum.

Step 3: Defects(s) in the fibromuscular layer of the posterior vaginal wall (if relevant) are identified.

Step 4: The fibromuscular layer of the posterior vaginal wall is plicated across the midline and/or site-specific defects in this layer is repaired with suture. This step must be performed cephalad to the hymenal ring. If a graft is being used, midline plication and/or sitespecific repair with sutures is optional. The graft is trimmed if indicated and attached as desired to the fibromuscular layer of the posterior vaginal wall or surrounding structures. The graft may be placed in a plane anterior to, posterior to, or within the fibromuscular layer of the posterior vaginal wall.

Step 5: The vaginal epithelium is closed over the repair.

Technique variations Possible variations described in the literature include the extent of the vaginal incision, the layer(s) of dissection (above the fibromuscular layer, splitting the thickness of the fibromuscular layer, or removal the fibromuscular layer off the rectum), the use of nonabsorbable or delayed absorbable suture, different suture gauges ( 0 or $2-0)$, the number of sutures used, plication of the fibromuscular layer across the midline alone and/or repair of specific defect(s) ("site-specific posterior repair") [134], and use of running or interrupted sutures for plication [135].

Variations of posterior vaginal repair with graft include different vaginal incisions, the layer(s) of dissection, use of nonabsorbable or absorbable sutures to fix the graft, whether or not underlying sutures in the fibromuscular layer are placed for plication and/or site-specific correction of defects, the number of graft arms or fixation points necessary to stabilize the augmented material, and the number of fixation points for the posterior graft. Graft attachment is ideally as close to the vaginal apex as possible in the cephalad direction, fixed laterally to the levator ani fascia, and fixed inferiorly to the perineal body, but different fixation points and sizes exist for different 
Fig. 13 Posterior vaginal repair

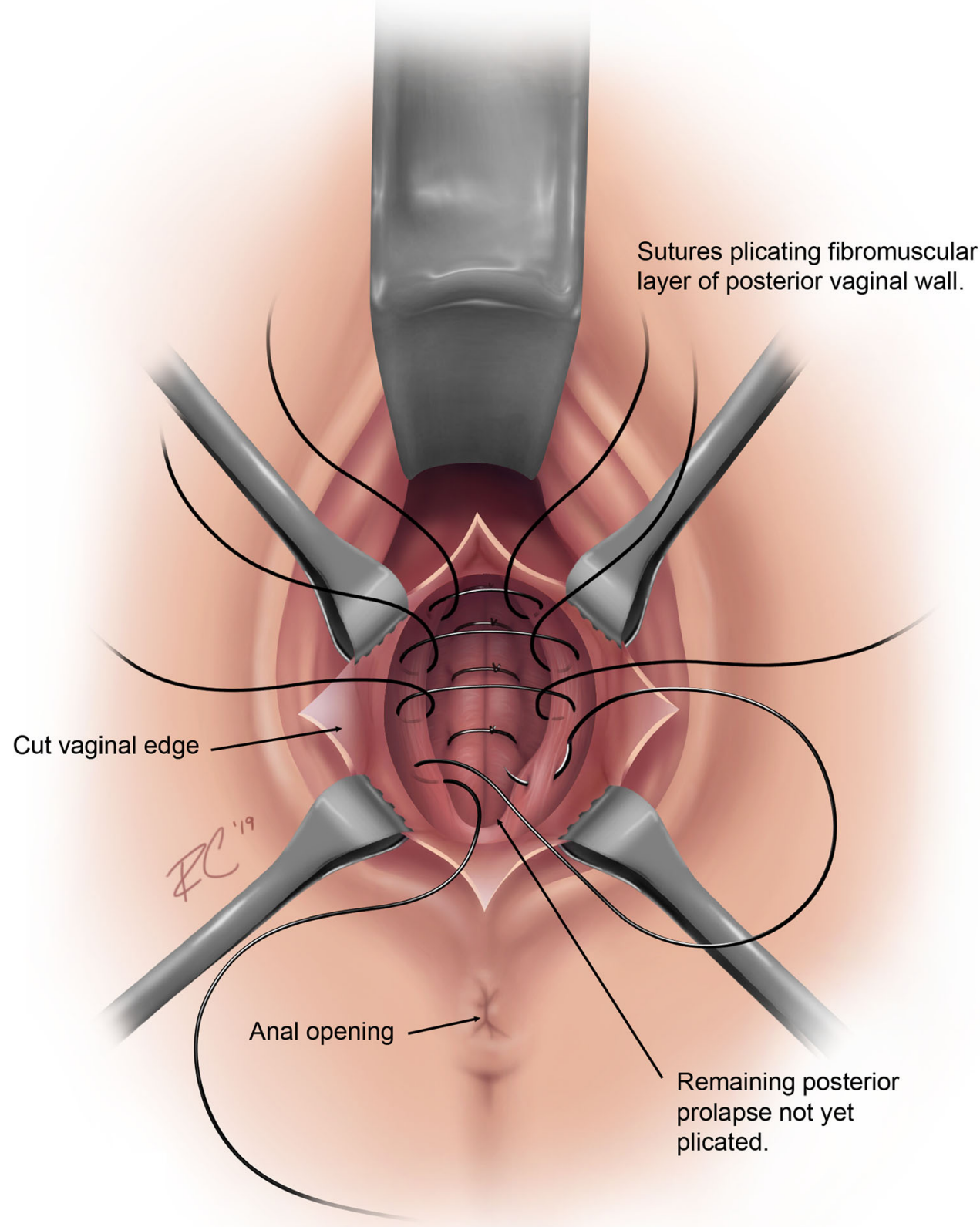

graft types. The most commonly described forms of graft materials are as follows: biologic graft (dermal, porcine submucosal), absorbable mesh (polyglactin, polydioxanone), nonabsorbable synthetic mesh (polypropylene), or composite (eg, polyglactin-polypropylene).

In posterior vaginal repair with graft, full-thickness dissection in the space between the vaginal epithelium and the fibromuscular layer of the posterior vaginal wall (as opposed to split thickness as often used in posterior vaginal repair without graft) often is described to theoretically reduce the risk of graft exposure. However, the depth of dissection or the layer in which the graft is placed would not change the terminology. Suturing of the graft into place is typically described as tension free, but the surgeon may modify the tension as clinically indicated without changing the terminology. 


\section{Special terminology considerations}

As all techniques of posterior vaginal repair are performed in extremely close approximation to the rectum, the procedure may be mistaken for procedures to support the rectum (eg, rectopexy) or for reconstruction of the anal sphincter (eg, anal sphincteroplasty). As these are not vaginal prolapse indications, confusing terms, such as "rectal repair," "anal repair," or "rectocele repair," are discouraged.

\section{Summary}

Posterior vaginal repairs use midline fibromuscular plication, which defines the subterm posterior colporrhaphy, and/or site-specific fibromuscular repair, which defines the subterm site-specific posterior vaginal repair. Posterior vaginal repair with graft carries the same definition as posterior vaginal without graft with the additional criteria that some type of graft material must be integrated into the posterior vaginal repair.

\section{Perineal repair}

A perineal repair is defined as approximation of the muscular tendon components of the perineal body back into the perineal body (Table 1, Fig. 14). The components approximated can include the deep and superficial transverse perineal muscles, the distal end of the fibromuscular layer of the posterior vaginal wall, the bulbospongiosus muscles, the anterior fibers of the external anal sphincter or its capsule, the puborectalis muscle, which contributes fibers to the superior external anal sphincter, and/or the perineal membrane. A perineal repair incorporates structures that are caudad to the hymenal remnant, in contrast to a posterior vaginal repair, which involves structures that are more proximal or cephalad to the hymenal remnant. The perineal body extends 2 - to $3-\mathrm{cm}$ proximal/ cephalad to the hymenal ring and is attached laterally to the puborectalis muscle. The aim of perineal repair is to narrow the genital hiatus and lengthen the perineal body to provide distal support to the vagina [136].

Perineal repair is historically performed at the end of various other prolapse repairs, especially posterior vaginal repair or colpocleisis; in most textbooks, this procedure is not described separately from the others. However, identification of this surgical term as an individual procedure is important in operative terminology.

\section{Procedure description}

Equipment A perineal repair can be approached transvaginally or transperineally, and basic equipment is the same as for other vaginal prolapse surgeries (Table 2).

\section{List of steps}

Step 1: A perineal repair is started by identifying the area of attenuation and the area of vaginal epithelium that needs to be excised. This is performed with Allis clamps at varying distances right and left of midline at the level of the hymen.

Step 2: A diamond- or sickle-shaped incision is made to remove a portion of distal vaginal epithelium and perineal skin. The underlying fibromuscular tissue may be undermined from the vaginal epithelium as well.

Step 3: Sutures are placed to approximate the selected perineal body components.

Step 4: Optional closure of the vaginal epithelium overlying these deep sutures.

Technique variations The starting point of the surgery may be the hymenal ring or the introitus, defined as where the line of the labia minora meets the perineum [137]. The extent of skin incision and dissection varies according to the extent of the defect [138]. There are variations in suture type and gauge [136]. The order of perineal muscle approximation can vary and may be performed individually or en bloc [136]. The approximated perineal body muscles can be attached superiorly to the levator ani muscles and/or the fibromuscular layer of the posterior vaginal wall [136].

\section{Special terminology considerations}

If the surgeon is performing a posterior vaginal repair at the same time, which is common, the terms "colpoperineorrhaphy" or "colpoperineoplasty" to designate the performance of both procedures should be avoided as it does not clearly delineate the posterior vaginal repair and the perineal repair. The term "perineoplasty" is not acceptable for perineal repair, as the term "perineoplasty" has been used interchangeably with widening genital hiatus for vaginal stenosis [139]. However, the synonym "perineorrhaphy" is acceptable as this term more specifically describes the nature of perineal repair.

In communications regarding this procedure, the specific components of the perineal body that the surgeon integrated should be specified, as the term perineal repair, while most appropriate, is not specific to which muscular or structural components are integrated by the surgeon. These details may be important in ongoing care of the patient or in the management of complications.

\section{Summary}

Perineal repair is performed to address a defect or attenuation of the perineal body, and it must approximate muscles of the 
perineal body. It generally results in a decrease in the size of the genital hiatus and an increase in the length of the perineal body.

\section{Levator ani plication}

Levator ani plication involves plication of the levator ani muscle toward the midline, incorporating a portion of the lateral fibromuscular layer of the posterior vaginal wall (Table 1) [140]. It is considered a constricting surgical procedure in which the goal is to narrow the distal vagina to close a defect in the genital hiatus. It is relatively contraindicated in women who have or desire future receptive vaginal intercourse because of high rates of dyspareunia.

\section{Procedure description}

Equipment A levator plication can be performed transperineally or transvaginally, and basic equipment matches these approaches (Table 2).

\section{List of steps}

Step 1: The distal portion of the levator ani muscles is identified either before or after the repair of the fibromuscular tissue in a posterior vaginal repair.

Step 2: Sutures are placed laterally in the levator ani muscles incorporating a portion of the lateral fibromuscular layer of the posterior vaginal wall.

Step 3: The sutures are tied in the midline to draw the muscles together to create a muscular shelf.

Technique variations Variations include the direction of suture placement to approximate the levator muscles and how many sutures or what suture types are placed. Variation exists in closure of vaginal or perineal epithelium over the muscle approximation sutures; some surgeons do not close this at all, some do only if needed for hemostasis, and some routinely close the epithelium.

\section{Special terminology considerations}

Even if the appropriate term is being used in communications about this procedure, the number of sutures and type of suture should be described in the operative report, as this may inform subsequent care. "Levatorpexy" is not an acceptable term, but the terms levatorrhaphy or levator myorrhaphy are acceptable if not ideal, as they describe the anatomy and procedure accurately.

\section{Summary}

Levator ani plication is performed to narrow the vaginal caliber by approximating the anterior levator ani muscles with sutures to create a distal muscular shelf.

\section{Obliterative procedures}

Obliterative procedures for POP, often termed colpocleisis, are defined as obliteration of the vaginal canal by removal of vaginal epithelium on the anterior and posterior vaginal walls and suturing together the fibromuscular layers of the anterior and posterior vaginal walls (Fig. 15). These procedures have high anatomic success, low rates of complications, and high patient satisfaction [141-145]. Obliterative procedures can be performed with or without hysterectomy where the uterus is in place or performed in the setting of a prior hysterectomy (Table 1). Procedures leaving the uterus in place, such as the eponymous LeFort colpocleisis, often involve the creation of bilateral epithelialized tunnels from the cervix to the introitus.

A surgeon in Metz, France, first suggested the idea of colpocleisis in 1823. Nearly 40 years later, in 1867, L. Neugebauer of Warsaw, Poland, performed the first procedure successfully. Over a century later, L. Le Fort of Paris popularized the procedure in 1977 with a recommendation of a broader surface denudation before suturing the anterior and posterior vaginal fibromuscular layers together. In the United States, colpocleisis with uterine preservation is still known as a "LeFort" procedure, and in other European Union countries, it is known as "Neugebauer-Le Fort." Although Le Fort did not originally include a concomitant hysterectomy with colpocleisis, 20th century advances in anesthesia allowed surgeons to include hysterectomy. The addition of hysterectomy was popularized to reduce the potential risk of future endometrial or cervical cancer [141, 146].

\section{Procedure description}

Equipment These procedures are always approached vaginally and require minimal instrumentation without the need for long instruments or lighting for deep spaces. Some surgeons use synthetic of biological graft between the anterior and posterior vaginal layers [147]. If tunnels are being created in a colpocleisis without hysterectomy, a vessel loop or small catheter may be used to ensure patency [148].

\section{List of steps}

Step 1: The vaginal epithelium is dissected off the underlying fibromuscular layers anteriorly and posteriorly, with or without leaving epithelial strips for later creation of tunnels for drainage.

Step 2: Multiple layers of sutures are placed plicating the posterior to the anterior fibromuscular layer of the 
Fig. 14 Perineal repair

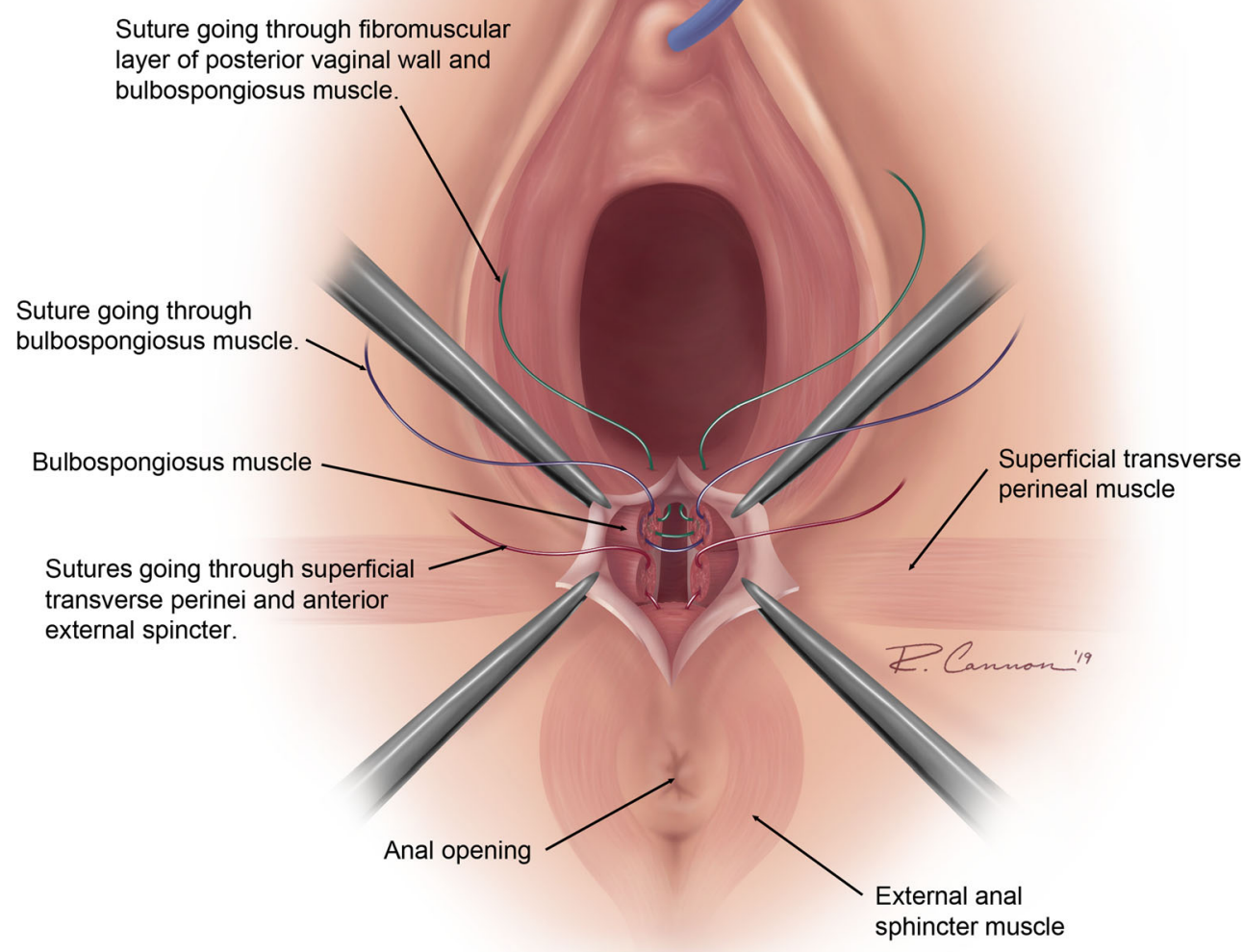

vaginal walls, either with purse-string or horizontal rows of interrupted sutures as appropriate.

Step 3: The vaginal epithelium on the distal edge of the repair is closed to make a new vaginal apex within a few centimeters of the hymen [144, 149-152].

Technique variations The decision to perform a hysterectomy at the time of colpocleisis should be individualized, and terminology used should specify if the uterus was present at the outset of the procedure, left in place, or if the colpocleisis was performed on a vaginal vault after a prior hysterectomy.

For a colpocleisis without hysterectomy (sometimes referred to as a modification of the Le Fort procedure), the anterior vaginal epithelium is removed proximal/cephalad to the urethrovesical junction to the cervix and the posterior vaginal epithelium is removed from the hymen to the cervix leaving at least 3-cm strips of intact vaginal epithelium along the lateral sides of the vagina. These remaining epithelial strips are used to create bilateral, epithelialized vaginal tunnels for potential uterine or cervical fluid drainage. The remaining fibromuscular layers of the anterior and posterior vaginal walls are then brought together with sequential rows of absorbable suture. A colpocleisis in the setting of either a prior hysterectomy or with a concurrent hysterectomy involves a more extensive denudation of the anterior and posterior vaginal epithelium without the creation of tunnels. It also involves approximation of the anterior and posterior fibromuscular layers in sequential sutures (either purse-string or horizontal rows).

\section{Special terminology considerations}

Extensive perineal repair and/or levator plication are often performed concurrently with colpocleisis to decrease the size 
Fig. 15 Colpocleisis

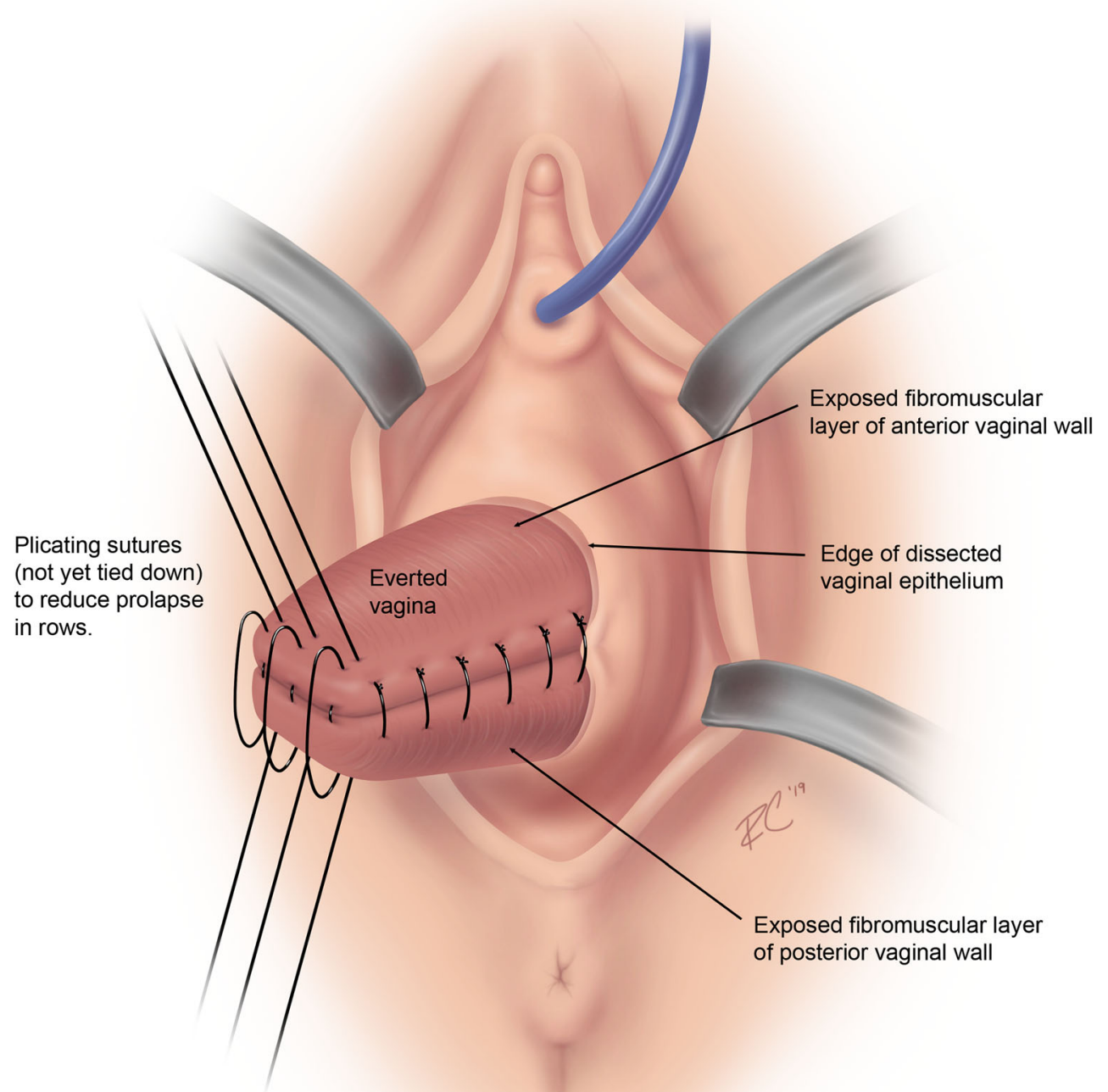

of the genital hiatus, as colpocleisis patients typically do not desire future coital activity. However, the use of the term "colpocleisis" does not automatically imply a levator plication and/or a perineal repair, so if these are performed, they must be specified as separate procedures. Some surgeons opt to perform endometrial sampling before performance of colpocleisis without hysterectomy, but this procedure is not assumed when the term colpocleisis is used, so surgeons must specify if dilation and curettage are performed at the same time.

\section{Summary}

Obliterative procedures result in a narrow, short vagina without an internal opening when the uterus is absent (colpocleisis with hysterectomy or colpocleisis of vaginal vault). In colpocleisis without hysterectomy where the uterus is left in place (Le Fort variation), tunnels for uterine or cervical drainage are ensured. Colpocleisis is always accomplished by some type of attachment of the fibromuscular layers of the anterior and posterior vaginal walls to one another.

\section{Discussion and recommendations}

The joint writing group of AUGS and IUGA concludes that surgical procedures to address POP require standardized definitions and have presented in this document relevant surgical terminology with detailed discussion of the associated surgery. This standardized terminology was developed, where possible, to 
include wording that highlights anatomical structures involved in the procedures and is specific enough not to be confused with other procedures for POP for which standardized terms have been developed. Conversely, the terminology in this document was developed to allow for some appropriate variations in the surgical steps, which we have outlined above.

It should be noted that, in general, discussion of a term in this document is neither an endorsement of that procedure nor intended to be a comprehensive discussion of either all the variations that have and could arise in the future. In addition, definition of a term in this document does not signify that the required procedural steps cover all procedures that should or could be performed concurrently for POP or other indications. For example, it is recommended to perform cystoscopy and a digital rectal examination after completion of most POP repair surgeries, but correct use of a surgical term from this document would not automatically indicate that cystoscopy and rectal examination were performed and had normal findings. Therefore, surgeons must be clear in communications that these recommended adjunct steps were completed and note the resultant findings.

We recommend that this terminology be used in communications regarding patient care, research, academic discussion, and medical oversight (including tracking of quality metrics and productivity for individual surgeons or medical groups). Furthermore, it would be ideal for the medical profession to integrate these recommendations into medical terminology around billing, coding, and financial tracking in the future, as this could avoid further miscommunication. Acknowledgment of these standardized terms in written academic publications related to female pelvic floor disorders should be indicated in the section Methods and Materials, or its equivalent, to read as follows: "Methods, definitions and units conform to the standards jointly recommended by the American Urogynecologic Society and the International Urogynecological Association, except where specifically noted."

The writing group's aim is that this terminology will allow sufficient clarity to communication of POP repair surgeries while still allowing sufficient variation and flexibility in an evolving surgical field.

Acknowledgements The following are being recognized for their contributions to this article: Kate V. Meriwether, MD (University of New Mexico, Albuquerque, NM); Karen P. Gold, MD MSCI (University of Oklahoma, School of Community Medicine, OK); Renaud de Tayrac, MD (Service de Gynécologie-Obstétrique, CHU Carémeau, Nîmes Cedex 9, France); Sara B. Cichowski, MD (Oregon Health Sciences University, Portland, OR); Vatche A. Minassian, MD, MPH (Brigham and Women's Hospital, Boston, MA); Rufus Cartwright, MD (Res), PhD (Oxford University Hospitals NHS Trust, London, United Kingdom); Pawel Miotla, MD, PhD (Medical University of Lublin, 2nd Department of Gynaecology, Lublin, Poland); Cara L. Grimes, MD, MAS (New York Medical College, Valhalla, NY); Luiz G.O. Brito,
MD, PhD (Department of Obstetrics and Gynecology, State University of Campinas, Campinas, Brazil); Tony M. Bazi, MD (American University of Beirut Medical Center, Beirut, Lebanon); Cassandra L. Carberry, MD (Alpert Medical School of Brown University/Women and Infants Hospital, Providence, RI); Lan Zhu, MD (Peking Union Medical College, Dong Dan, Dongcheng, Beijing, China); and Rebecca G. Rogers, MD (University of Texas at Austin, Dell Medical School, Austin, TX)

\section{Compliance with ethical standards}

Conflict of interest K.V.M. is a voting board member for Society of Gynecologic Surgeons with travel stipend and received book editor royalties from Elsevier publishing. R.G.R. received royalties from Uptodate and stipend and travel from the American Board of Obstetrics and Gynecology (ABOG) and International Urogynecologic Association (IUGA). C.L.G. is an expert witness for Johnson \& Johnson. R. D. is a consultant for Boston Scientific (personnel fees and research grant) and for Coloplast (research grant) and received travel fund from the European Urogynaecological Association (EUGA). The other contributors have declared that there are no conflicts of interest.

\section{Appendix 1: Future Directions of selected procedures}

Sacrocolpopexy:

The use of tacking devices to complete an all-vaginal approach [153], as well as transvaginal single-port laparoscopic SCP with the graft attached to the sacrum with suture, have been described $[154,155]$. Fixation of the graft to the anterior and posterior vagina with adhesive at the time of SCP has been described and found to decrease the time of procedure [156-158].

Uterosacral ligament suspension:

These techniques have remained largely unchanged since their original description, but future directions could include improved suture materials, devices for USL tenting and suture placement in the same orientation, or improved lighting devices for better anatomical visualization.

Sacrospinous ligament fixation:

Similar to USLS, this procedure remains mostly unchanged since its initial description with the exception of newer needle passage devices. Future directions could include improved suture materials, more ergonomic or endoscopic linked suture passage devices, or improved lighting devices for better anatomical visualization.

Hysteropexy procedures:

The future of hysteropexy will likely involve development of natural orifice transluminal endoscopic surgery (NOTES) or graft fixation technology [159] to allow for less morbidity and increased durability of nonabsorbable graft use in approach to SHP [86, 93].

Anterior and posterior vaginal wall repairs:

Tissue culture engineering may aid in the creation of a biologically based graft that has ideal characteristics for implantation in anterior or posterior vaginal wall repairs. For 
posterior repair, some experimental studies with endometrial mesenchymal stem cells as targeted therapy for POP were published; however, human studies are needed [160]. To date, there is no innovative technology that has demonstrated improved clinical outcomes over native tissue repairs in the posterior compartment [127, 161-163], so the use of augmentation materials in prolapse surgery has been under scrutiny, and at present, studies in this area are not encouraging. The use of mesenchymal cells with synthetic grafts has been tested to improve use of the material, but no clinical evidence of better results to this moment [164].

\section{Appendix 2: Transvaginal mesh procedures for POP in 2019}

Certain prefabricated transvaginal grafts, which were available in early 2019 , were withdrawn from the marketplace following an April 2019 order from the US FDA for "manufacturers of all remaining surgical mesh products indicated for the transvaginal repair of pelvic organ prolapse to stop the sale and distribution their products in the US immediately." [165] This news release followed a convening of an advisory committee meeting "on the issue to share available evidence and seek expert opinion on how to evaluate the risks and benefits of these devices." Specifically, they determined in their advisory committee that the manufacturers still with products on the market had not adequately demonstrated "reasonable assurance" that these devices had safety and effectiveness, which is the standard for class 3 surgical devices. Surgical mesh products for transvaginal repair of POP have been class 3 products since their reclassification by the FDA in January of 2016.

\section{References}

1. Wu JM, Dieter AA, Pate VJ, et al. Cumulative incidence of a subsequent surgery after stress urinary incontinence and pelvic organ prolapse procedure. Obstet Gynecol. 2017;129(6):1124-30.

2. Haylen BT, Freeman RM, Swift SE, et al. An International Urogynecological Association (IUGA) / International Continence Society (ICS) joint terminology and classification of the complications related directly to the insertion of prostheses (meshes, implants, tapes) \& grafts in female pelvic floor surgery. Int Urogynecol J. 2011;22(1):3-15.

3. Toozs-Hobson P, Freeman R, Barber M, et al. An International Urogynecological Association (IUGA)/International Continence Society (ICS) joint report on the terminology for reporting outcomes of surgical procedures for pelvic organ prolapse. Int Urogynecol J. 2012;23(5):527-35.

4. Haylen BT, Maher CF, Barber MD, et al. An International Urogynecological Association (IUGA) / International Continence Society (ICS) joint report on the terminology for female pelvic organ prolapse (POP). Int Urogynecol J. 2016;27(2): 165-94.

5. Jeppson PC, Balgobin S, Washington BB, et al. Recommended standardized terminology of the anterior female pelvis based on a structured medical literature review. Am J Obstet Gynecol. 2018;219(1):26-39.

6. Bump RC, Mattiasson A, Bo K, et al. The standardization of terminology of female pelvic organ prolapse and pelvic floor dysfunction. Am J Obstet Gynecol. 1996;175(1):10-7.

7. Lane FE. Repair of posthysterectomy vaginal-vault prolapse. Obstet Gynecol. 1962;20:72-7.

8. Arthure HG, Savage D. Uterine prolapse and prolapse of the vaginal vault treated by sacral hysteropexy. J Obstet Gynaecol Br Emp. 1957;64(3):355-60.

9. Abernethy M, Vasquez E, Kenton K, et al. Where do we place the sacrocolpopexy stitch? A magnetic resonance imaging investigation. Female Pelvic Med Reconstr Surg. 2013;19(1):31-3.

10. Good MM, Abele TA, Balgobin S, et al. Preventing L5-S1 discitis associated with sacrocolpopexy. Obstet Gynecol. 2013;121(2 Pt 1):285-90.

11. Amid P. Classification of biomaterials and their related complications in abdominal wall surgery. Hernia. 1997;1(1):15-21.

12. Illiano E, Ditonno P, Giannitsas K, et al. Robot-assisted versus laparoscopic sacrocolpopexy for high-stage pelvic organ prolapse: a prospective, randomized, single-center study. Urology. 2019;134(134):116-23.

13. Brubaker L, Nygaard I, Richter HE, et al. Two-year outcomes after sacrocolpopexy with and without burch to prevent stress urinary incontinence. Obstet Gynecol. 2008;112(1):49-55.

14. Costantini E, Mearini L, Lazzeri M, et al. Laparoscopic versus abdominal sacrocolpopexy: a randomized, controlled trial. J Urol. 2016;196(1):159-65.

15. Snyder TE, Krantz KE. Abdominal-retroperitoneal sacral colpopexy for the correction of vaginal prolapse. Obstet Gynecol. 1991;77(6):944-9.

16. Addison WA, Timmons MC, Wall LL, et al. Failed abdominal sacral colpopexy: observations and recommendations. Obstet Gynecol. 1989;74(3 Pt 2):480-3.

17. Nygaard IE, McCreery R, Brubaker L, et al. Abdominal sacrocolpopexy: a comprehensive review. Obstet Gynecol. 2004;104(4):805-23.

18. Maher C, Feiner B, Baessler K, et al. Surgery for women with apical vaginal prolapse. Cochrane Database Syst Rev. 2016;10: CD012376.

19. Whitehead WE, Bradley CS, Brown MB, et al. Pelvic Floor Network. Gastrointestinal complications following abdominal sacrocolpopexy for advanced pelvic organ prolapse. Am J Obstet Gynecol. 2007;197(1):78.e71-7.

20. Lansman HH. Posthysterectomy vault prolapse: sacral colpopexy with dura mater graft. Obstet Gynecol. 1984;63(4):577-82.

21. Hendee AE, Berry CM. Abdominal sacropexy for vaginal vault prolapse. Clin Obstet Gynecol. 1981;24(4):1217-26.

22. Salamon CG, Lewis C, Priestley J, et al. Prospective study of an ultra-lightweight polypropylene $\mathrm{Y}$ mesh for robotic sacrocolpopexy. Int Urogynecol J. 2013;24(8):1371-5.

23. Culligan PJ, Salamon C, Priestley JL, et al. Porcine dermis compared with polypropylene mesh for laparoscopic sacrocolpopexy: a randomized controlled trial. Obstet Gynecol. 2013;121(1):14351.

24. Nosti PA, Carter CM, Sokol AI, et al. Transvaginal versus transabdominal placement of synthetic mesh at time of sacrocolpopexy. Female Pelvic Med Reconstr Surg. 2016;22(3): $151-5$.

25. Cundiff GW, Harris RL, Coates $\mathrm{K}$, et al. Abdominal sacral colpoperineopexy: a new approach for correction of posterior compartment defects and perineal descent associated with vaginal vault prolapse. Am J Obstet Gynecol. 1997;177(6):1345-53.

26. Grimes CL, Quiroz LH, Gutman RE, et al. Long-term impact of abdominal sacral colpoperineopexy on symptoms of obstructed defecation. Female Pelvic Med Reconstr Surg. 2010;16(4):234-7. 
27. Link RE, Su LM, Bhayani SB, et al. Laparoscopic sacral colpoperineopexy for treatment of perineal body descent and vaginal vault prolapse. Urology. 2004;64(1):145-7.

28. Rosenblatt PL, Chelmow D, Ferzandi TR. Laparoscopic sacrocervicopexy for the treatment of uterine prolapse: a retrospective case series report. J Minim Invasive Gynecol. 2008;15(3):268-72.

29. van Zanten F, Schraffordt Koops SE, O'Sullivan OE, et al. Robotassisted surgery for the management of apical prolapse: a bicentre prospective cohort study. BJOG. 2019;126(8):1065-73.

30. Bataller E, Ros C, Angles S, et al. Anatomical outcomes 1 year after pelvic organ prolapse surgery in patients with and without a uterus at a high risk of recurrence: a randomised controlled trial comparing laparoscopic sacrocolpopexy/cervicopexy and anterior vaginal mesh. Int Urogynecol J. 2019;30(4):545-55.

31. Brubaker L, Cundiff GW, Fine P, et al. Abdominal sacrocolpopexy with Burch colposuspension to reduce urinary stress incontinence. N Engl J Med. 2006;354(15):1557-66.

32. Miller N. A new method of correcting complete inversion of the vagina: with or without complete prolapse; report of two cases. Surg Gynecol Obstet. 1927:550-5.

33. Webb MJ, Aronson MP, Ferguson LK, et al. Posthysterectomy vaginal vault prolapse: primary repair in 693 patients. Obstet Gynecol. 1998;92(2):281-5.

34. Rardin CR, Erekson EA, Sung VW, et al. Uterosacral colpopexy at the time of vaginal hysterectomy: comparison of laparoscopic and vaginal approaches. J Reprod Med. 2009;54(5):273-80.

35. Houlihan S, Kim-Fine S, Birch C, et al. Uterosacral vault suspension (USLS) at the time of hysterectomy: laparoscopic versus vaginal approach. Int Urogynecol J. 2019;30(4):611-21.

36. McCall ML. Posterior culdeplasty; surgical correction of enterocele during vaginal hysterectomy; a preliminary report. Obstet Gynecol. 1957;10(6):595-602.

37. Richardson AC, Lyon JB, Williams NL. A new look at pelvic relaxation. Am J Obstet Gynecol. 1976;126(5):568-73.

38. Shull BL, Bachofen C, Coates KW, et al. A transvaginal approach to repair of apical and other associated sites of pelvic organ prolapse with uterosacral ligaments. Am J Obstet Gynecol. 2000;183(6):1365-73.

39. Barber MD, Visco AG, Weidner AC, et al. Bilateral uterosacral ligament vaginal vault suspension with site-specific endopelvic fascia defect repair for treatment of pelvic organ prolapse. Am J Obstet Gynecol. 2000;183(6):1402-10.

40. Jelovsek JE, Barber MD, Brubaker L, et al. Effect of uterosacral ligament suspension vs sacrospinous ligament fixation with or without perioperative behavioral therapy for pelvic organ vaginal prolapse on surgical outcomes and prolapse symptoms at 5 years in the OPTIMAL randomized clinical trial. JAMA. 2018;319(15): 1554-65.

41. Restaino S, Ronsini C, Finelli A, et al. Laparoscopic approach for Shull repair of pelvic floor defects. J Minim Invasive Gynecol. 2018;25(6):954.

42. Panico G, Campagna G, Caramazza D, et al. Laparoscopic high uterosacral ligament suspension: an alternative route for a traditional technique. Int Urogynecol J. 2018;29(8):1227-9.

43. Cohen SA, Carberry CL, Smilen SW. American Urogynecologic Society Consensus Statement: cystoscopy at the time of prolapse repair. Female Pelvic Med Reconstr Surg. 2018;24(4):258-9.

44. Jenkins VR 2nd. Uterosacral ligament fixation for vaginal vault suspension in uterine and vaginal vault prolapse. Am J Obstet Gynecol. 1997;177(6):1337-43.

45. Karram M, Goldwasser S, Kleeman S, et al. High uterosacral vaginal vault suspension with fascial reconstruction for vaginal repair of enterocele and vaginal vault prolapse. Am J Obstet Gynecol. 2001;185(6):1339-42.
46. Spelzini F, Frigerio M, Manodoro S, et al. Modified McCall culdoplasty versus Shull suspension in pelvic prolapse primary repair: a retrospective study. Int Urogynecol J. 2017;28(1):65-71.

47. Fatton B, Dwyer PL, Achtari C, et al. Bilateral extraperitoneal uterosacral vaginal vault suspension: a 2-year follow-up longitudinal case series of 123 patients. Int Urogynecol J. 2009;20(4): 427-34.

48. Karmakar D, Dwyer PL, Thomas E, Schierlitz L. Extraperitoneal uterosacral suspension technique for post hysterectomy apical prolapse in 472 women: results from a longitudinal clinical study. BJOG. 2019;126(4):536-42.

49. Unger CA, Walters MD, Ridgeway B, Jelovsek JE, Barber MD, Paraiso MF. Incidence of adverse events after uterosacral colpopexy for uterovaginal and posthysterectomy vault prolapse. Am J Obstet Gynecol. 2015;212(5):603.e601-7.

50. Sagsoz N, Ersoy M, Kamaci M, Tekdemir I. Anatomical landmarks regarding sacrospinous colpopexy operations performed for vaginal vault prolapse. Eur J Obstet Gynecol Reprod Biol. 2002;101(1):74-8

51. Sederl J. Surgery in prolapse of a blind-end vagina [in German] Geburtshilfe Frauenheilkd. 1958;18(6):824-8.

52. Richter $\mathrm{K}$. The surgical anatomy of the vaginaefixatio sacrospinalis vaginalis. A contribution to the surgical treatment of vaginal blind pouch prolapse [in German]. Geburtshilfe Frauenheilkd. 1968;28(4):321-7.

53. Wang Y, Wang D, Li Y, et al. Laparoscopic sacrospinous ligament fixation for uterovaginal prolapse: experience with 93 cases. Int Urogynecol J. 2011;22(1):83-9.

54. Randall CL, Nichols DH. Surgical treatment of vaginal inversion. Obstet Gynecol. 1971;38(3):327-32.

55. Larson KA, Smith T, Berger MB, et al. Long-term patient satisfaction with Michigan four-wall sacrospinous ligament suspension for prolapse. Obstet Gynecol. 2013;122(5):967-75.

56. Manning JA, Arnold P. A review of six sacrospinous suture devices. Aust N Z J Obstet Gynaecol. 2014;54(6):558-63.

57. Cespedes RD. Anterior approach bilateral sacrospinous ligament fixation for vaginal vault prolapse. Urology. 2000;56(6 suppl 1): $70-5$.

58. Leone Roberti Maggiore U, Alessandri F, Remorgida V, Venturini PL, Ferrero S. Vaginal sacrospinous colpopexy using the Capio suture-capturing device versus traditional technique: feasibility and outcome. Arch Gynecol Obstet. 2013;287(2):267-74.

59. Mowat A, Wong V, Goh J, et al. A descriptive study on the efficacy and complications of the Capio (Boston Scientific) suturing device for sacrospinous ligament fixation. Aust N Z J Obstet Gynaecol. 2018;58(1):119-24.

60. Lo TS, Horng SG, Huang HJ, et al. Repair of recurrent vaginal vault prolapse using sacrospinous ligament fixation with mesh interposition and reinforcement. Acta Obstet Gynecol Scand. 2005;84(10):992-5.

61. Seitz M, Jirschele K, Tran A, Iyer S, Gafni-Kane A, Tomezsko J, et al. A comparison of sacrospinous hysteropexy augmented with polypropylene mesh versus human dermis at 12-month follow-up: an ambidirectional study. Female Pelvic Med Reconstr Surg. 2018:1. https://doi.org/10.1097/SPV.0000000000000640.

62. Jirschele K, Seitz M, Zhou Y, Rosenblatt P, Culligan P, Sand P. A multicenter, prospective trial to evaluate mesh-augmented sacrospinous hysteropexy for uterovaginal prolapse. Int Urogynecol J. 2015;26(5):743-8.

63. Geoffrion R, Hyakutake MT, Koenig NA, et al. Bilateral sacrospinous vault fixation with tailored synthetic mesh arms: clinical outcomes at one year. J Obstet Gynaecol Can. 2015;37(2):129-37.

64. Rahkola-Soisalo P, Mikkola TS, Altman D, et al. Pelvic organ prolapse repair using the uphold vaginal support system: 5-year follow-up. Female Pelvic Med Reconstr Surg. 2019;25(3):200-5. 
65. Scotti RJ, Garely AD, Greston WM, Flora RF, Olson TR. Paravaginal repair of lateral vaginal wall defects by fixation to the ischial periosteum and obturator membrane. Am J Obstet Gynecol. 1998;179(6 Pt 1):1436-45.

66. Kearney R, DeLancey JO. Selecting suspension points and excising the vagina during Michigan four-wall sacrospinous suspension. Obstet Gynecol. 2003;101(2):325-30.

67. Lo TS, Akhtar Bt Nawawi E, Al-Kharabsheh AM. Anterior approach unilateral right sacrospinous fixation for recurrent pelvic organ prolapse following multiple colorectal surgeries. Int $\mathbf{J}$ Colorectal Dis. 2015;30(8):1141-2.

68. Serati M, Braga A, Bogani G, et al. Iliococcygeus fixation for the treatment of apical vaginal prolapse: efficacy and safety at 5 years of follow-up. Int Urogynecol J. 2015;26(7):1007-12.

69. Inmon WB. Pelvic relaxation and repair including prolapse of vagina following hysterectomy. South Med J. 1963;56:577-82.

70. Shull BL, Capen CV, Riggs MW, et al. Bilateral attachment of the vaginal cuff to iliococcygeus fascia: an effective method of cuff suspension. Am J Obstet Gynecol. 1993;168(6 Pt 1):1669-74.

71. Meeks GR, Washburne JF, McGehee RP, et al. Repair of vaginal vault prolapse by suspension of the vagina to iliococcygeus (prespinous) fascia. Am J Obstet Gynecol. 1994;171(6):1444-52.

72. Thornton WN Jr, Peters WA 3rd. Repair of vaginal prolapse after hysterectomy. Am J Obstet Gynecol. 1983;147(2):140-8.

73. Paz-Levy D, Yohay D, Neymeyer J, et al. Native tissue repair for central compartment prolapse: a narrative review. Int Urogynecol J. 2017;28(2):181-9.

74. Morley GW, DeLancey JO. Sacrospinous ligament fixation for eversion of the vagina. Am J Obstet Gynecol. 1988;158(4):87281.

75. Zhang QX, Lang JH, Zhu L, et al. Clinical anatomic study on ischial spinous fascia fixation [in Chinese]. Zhonghua $\mathrm{Fu}$ Chan Ke Za Zhi. 2009;44(5):350-3.

76. DeLancey JO. Anatomic aspects of vaginal eversion after hysterectomy. Am J Obstet Gynecol. 1992;166(6 Pt 1):1717-24.

77. Ross WT, Meister MR, Shepherd JP, et al. Utilization of apical vaginal support procedures at time of inpatient hysterectomy performed for benign conditions: a national estimate. Am J Obstet Gynecol. 2017;217(4):436.e431-8.

78. Meriwether KV, Antosh DD, Olivera CK, et al. Uterine preservation vs hysterectomy in pelvic organ prolapse surgery: a systematic review with meta-analysis and clinical practice guidelines. Am J Obstet Gynecol. 2018;219(2):129-146.e122.

79. Costantini E, Lazzeri M, Zucchi A, et al. Five-year outcome of uterus sparing surgery for pelvic organ prolapse repair: a singlecenter experience. Int Urogynecol J. 2011;22(3):287-92.

80. Roovers JP, van der Bom JG, van der Vaart CH, et al. A randomized comparison of post-operative pain, quality of life, and physical performance during the first 6 weeks after abdominal or vaginal surgical correction of descensus uteri. Neurourol Urodyn. 2005;24(4):334-40.

81. Veit-Rubin N, Dubuisson JB, Lange S, et al. Uterus-preserving laparoscopic lateral suspension with mesh for pelvic organ prolapse: a patient-centred outcome report and video of a continuous series of 245 patients. Int Urogynecol J. 2016;27(3):491-3.

82. Hsieh $\mathrm{CH}$. A new laparoscopic technique for uterine prolapse: one-sided uterine fixation through the round ligament. Int Urogynecol J. 2011;22(2):213-9.

83. Joshi VM, Otiv SR, Dagade VB, et al. Pectineal ligament hysteropexy for uterine prolapse in premenopausal women by open and laparoscopic approach in Indian urban and rural centers. Female Pelvic Med Reconstr Surg. 2015;21(4):215-9.

84. Khanam RA, Rubaiyat A, Azam MS. Sling for correcting uterine prolapse: twelve years experience. Mymensingh Med J. 2014;23(1):13-7.
85. Romanzi LJ, Tyagi R. Hysteropexy compared to hysterectomy for uterine prolapse surgery: does durability differ? Int Urogynecol J. 2012;23(5):625-31.

86. Kow N, Goldman HB, Ridgeway B. Uterine conservation during prolapse repair: 9-year experience at a single institution. Female Pelvic Med Reconstr Surg. 2016;22(3):126-31.

87. Bedford ND, Seman EI, O'Shea RT, et al. Effect of uterine preservation on outcome of laparoscopic uterosacral suspension. J Minim Invasive Gynecol. 2013;20(2):172-7.

88. Rosen DM, Shukla A, Cario GM, et al. Is hysterectomy necessary for laparoscopic pelvic floor repair? A prospective study. J Minim Invasive Gynecol. 2008;15(6):729-34.

89. Diwan A, Rardin CR, Strohsnitter WC, Weld A, Rosenblatt P, Kohli N. Laparoscopic uterosacral ligament uterine suspension compared with vaginal hysterectomy with vaginal vault suspension for uterovaginal prolapse. Int Urogynecol J Pelvic Floor Dysfunct. 2006;17(1):79-83.

90. Detollenaere RJ, den Boon J, Stekelenburg J, et al. Sacrospinous hysteropexy versus vaginal hysterectomy with suspension of the uterosacral ligaments in women with uterine prolapse stage 2 or higher: multicentre randomised non-inferiority trial. BMJ. 2015;351:h3717.

91. Dietz V, van der Vaart CH, van der Graaf Y, et al. One-year followup after sacrospinous hysteropexy and vaginal hysterectomy for uterine descent: a randomized study. Int Urogynecol J. 2010;21(2):209-16.

92. Khandwala S. Transvaginal mesh surgery for pelvic organ prolapse: one-year outcome analysis. Female Pelvic Med Reconstr Surg. 2013;19(2):84-9.

93. Gutman RE, Rardin CR, Sokol ER, et al. Vaginal and laparoscopic mesh hysteropexy for uterovaginal prolapse: a parallel cohort study. Am J Obstet Gynecol. 2017;216(1):38.e31-11.

94. Milani AL, Withagen MI, Vierhout ME. Outcomes and predictors of failure of trocar-guided vaginal mesh surgery for pelvic organ prolapse. Am J Obstet Gynecol. 2012;206(5):440.e1-8.

95. Oversand SH, Staff AC, Spydslaug AE, Svenningsen R, Borstad E. Long-term follow-up after native tissue repair for pelvic organ prolapse. Int Urogynecol J. 2014;25(1):81-9.

96. Conger GT, Keettel WC. The Manchester-Fothergill operation, its place in gynecology; a review of 960 cases at university hospitals, Iowa City, Iowa. Am J Obstet Gynecol. 1958;76(3):634-40.

97. Tipton RH, Atkin PF. Uterine disease after the Manchester repair operation. J Obstet Gynaecol Br Commonw. 1970;77(9):852-3.

98. Ayhan A, Esin S, Guven S, et al. The Manchester operation for uterine prolapse. Int J Gynaecol Obstet. 2006;92(3):228-33.

99. Szczesniewska A, Szpakowski M, Wladzinski J, et al. (UN)Forgotten Neugebauer-Le Fort operation. Paramedian closure of the vagina - safe and effective surgical procedure for treating of pelvic organ prolapse in older women. Ginekol Pol. 2015;86(3):198-202.

100. Gutman R, Maher C. Uterine-preserving POP surgery. Int Urogynecol J. 2013;24(11):1803-13.

101. Ameline A, Huguier J. Posterior suspension to the lumbo-sacral disk; abdominal method of replacement of the utero-sacral ligaments [in French]. Gynecol Obstet (Paris). 1957;56(1):94-8.

102. Huguier J, Scali P. Posterior suspension of the genital axis on the lumbosacral disk in the treatment of uterine prolapse [in French]. Presse Med. 1958;66(35):781-4.

103. Diwan A, Rardin CR, Kohli N. Uterine preservation during surgery for uterovaginal prolapse: a review. Int Urogynecol J Pelvic Floor Dysfunct. 2004;15(4):286-92.

104. Kow N, Goldman HB, Ridgeway B. Management options for women with uterine prolapse interested in uterine preservation. Curr Urol Rep. 2013;14(5):395-402.

105. Paek J, Lee M, Kim BW, et al. Robotic or laparoscopic sacrohysteropexy versus open sacrohysteropexy for uterus 
preservation in pelvic organ prolapse. Int Urogynecol J. 2016;27(4):593-9.

106. Pan K, Cao L, Ryan NA, et al. Laparoscopic sacral hysteropexy versus laparoscopic sacrocolpopexy with hysterectomy for pelvic organ prolapse. Int Urogynecol J. 2016;27(1):93-101.

107. Gracia M, Perello M, Bataller E, et al. Comparison between laparoscopic sacral hysteropexy and subtotal hysterectomy plus cervicopexy in pelvic organ prolapse: a pilot study. NeurourolUrodyn. 2015;34(7):654-8.

108. Veit-Rubin N, Dubuisson J, Constantin F, et al. Uterus preservation is superior to hysterectomy when performing laparoscopic lateral suspension with mesh. Int Urogynecol J. 2019;30(4):55764.

109. Rimailho J, Talbot C, Bernard JD, et al. Anterolateral hysteropexy via abdominal approach. Results and indications. Apropos of a series of 92 patients [in French]. Ann Chir. 1993;47(3):244-9.

110. Li S, Ji M, Zhao Z. The effectiveness of two different laparoscopic surgeries for apical support of pelvic organ prolapse. Eur J Obstet Gynecol Reprod Biol. 2015;188:74-8.

111. van Brummen HJ, van de Pol G, Aalders CI, Heintz AP, van der Vaart $\mathrm{CH}$. Sacrospinous hysteropexy compared to vaginal hysterectomy as primary surgical treatment for a descensus uteri: effects on urinary symptoms. Int Urogynecol J Pelvic Floor Dysfunct. 2003;14(5):350-5; discussion 355.

112. Dietz V, de Jong J, Huisman M, Schraffordt Koops S, Heintz P, van der Vaart $H$. The effectiveness of the sacrospinous hysteropexy for the primary treatment of uterovaginal prolapse. Int Urogynecol J Pelvic Floor Dysfunct. 2007;18(11):1271-6.

113. Florian-Rodriguez ME, Hare A, Chin K, et al. Inferior gluteal and other nerves associated with sacrospinous ligament: a cadaver study. Am J Obstet Gynecol. 2016;215(5):646.e1-6.

114. Weber AM, Walters MD. Anterior vaginal prolapse: review of anatomy and techniques of surgical repair. Obstet Gynecol. 1997;89(2):311-8.

115. Lensen EJ, Withagen MI, Kluivers KB, et al. Surgical treatment of pelvic organ prolapse: a historical review with emphasis on the anterior compartment. Int Urogynecol J. 2013;24(10):1593-602.

116. Halpern-Elenskaia K, Umek W, Bodner-Adler B, et al. Anterior colporrhaphy: a standard operation? Systematic review of the technical aspects of a common procedure in randomized controlled trials. Int Urogynecol J. 2018;29(6):781-8.

117. Shull BL, Benn SJ, Kuehl TJ. Surgical management of prolapse of the anterior vaginal segment: an analysis of support defects, operative morbidity, and anatomic outcome. Am J Obstet Gynecol. 1994;171(6):1429-36; discussion 1436-1429.

118. van Geelen JM, Dwyer PL. Where to for pelvic organ prolapse treatment after the FDA pronouncements? A systematic review of the recent literature. Int Urogynecol J Pelvic Floor Dysfunct. 2013;24(5):707-18.

119. Urogynecologic Surgical Mesh Implants. U.S. Food and Drug Administration. Available at: https://www.fda.gov/medicaldevices/implants-and-prosthetics/urogynecologic-surgical-meshimplants. Accessed July 3, 2019.

120. Lopes TSN, Hilton P, Monaghan J. Bonney's Gynaecological Surgery. 12th ed: Wiley-Blackwell; 2018; Hoboken, NJ. Pages: 161-192.

121. Baggish MKM. Atlas of pelvic anatomy and gynecologic surgery. 2nd ed: Saunders Elsevier; 2006.

122. Fairclough E, Myers J, Smith ARB, et al. A UK questionnaire survey of current techniques used to perform pelvic organ prolapse repair. Int Urogynecol J. 2017;28(9):1367-76.

123. White GR. Cystocele - a radical cure by suturing lateral sulci of the vagina to the white line of pelvic fascia. 1909. Int Urogynecol J Pelvic Floor Dysfunct. 1997;8(5):288-92.
124. Richardson AC, Edmonds PB, Williams NL. Treatment of stress urinary incontinence due to paravaginal fascial defect. Obstet Gynecol. 1981;57(3):357-62.

125. Scarpero HM, Cespedes RD, Winters JC. Transabdominal approach to repair of vaginal vault prolapse. Tech Urol. 2001;7(2): 139-45.

126. DeLancey JO. Structural anatomy of the posterior pelvic compartment as it relates to rectocele. Am J Obstet Gynecol. 1999;180(4): 815-23.

127. Paraiso MF, Barber MD, Muir TW, Walters MD. Rectocele repair: a randomized trial of three surgical techniques including graft augmentation. Am J Obstet Gynecol. 2006;195(6):1762-71.

128. Kim S, Pollock GR, Twiss CO, et al. Surgery for posterior compartment vaginal prolapse: graft augmented repair. Urol Clin North Am. 2019;46(1):87-95.

129. Jeffcoate TN. Posterior colpoperineorrhaphy. Am J Obstet Gynecol. 1959;77(3):490-502.

130. Richardson AC. The rectovaginal septum revisited: its relationship to rectocele and its importance in rectocele repair. Clin Obstet Gynecol. 1993;36(4):976-83.

131. Noe GK, Alkatout I, Schiermeier S, et al. Laparoscopic anterior and posterior native tissue repair: a new pelvic floor approach. Minim Invasive Ther Allied Technol. 2019;28(4):241-6.

132. Zimmermann EF, Hayes RS, Daniels IR, Smart NJ, Warwick AM. Transperineal rectocele repair: a systematic review. ANZ J Surg. 2017;87(10):773-9.

133. Karram M, Maher C. Surgery for posterior vaginal wall prolapse. Int Urogynecol J Pelvic Floor Dysfunct. 2013;24(11):1835-41.

134. Glavind K, Christiansen AG. Site-specific colporrhaphy in posterior compartment pelvic organ prolapse. Int Urogynecol J. 2016;27(5):735-9.

135. Schmidlin-Enderli K, Schuessler B. A new rectovaginal fascial plication technique for treatment of rectocele with obstructed defecation: a proof of concept study. Int Urogynecol J Pelvic Floor Dysfunct. 2013;24(4):613-9.

136. Kanter G, Jeppson PC, McGuire BL, et al. Perineorrhaphy: commonly performed yet poorly understood. A survey of surgeons. Int Urogynecol J. 2015;26(12):1797-801.

137. Haylen BT, Younis M, Naidoo S, et al. Perineorrhaphy quantitative assessment (Pe-QA). Int Urogynecol J. 2015;26(4):539-44.

138. Christmann-Schmid C, Wierenga AP, Frischknecht E, Maher C. A prospective observational study of the classification of the perineum and evaluation of perineal repair at the time of posterior colporrhaphy. Female Pelvic Med Reconstr Surg. 2016;22(6): 453-9.

139. Rouzier R, Haddad B, Deyrolle C, et al. Perineoplasty for the treatment of introital stenosis related to vulvar lichen sclerosus. Am J Obstet Gynecol. 2002;186(1):49-52.

140. Baggish M. Atlas of Pelvic Anatomy and Gynecologic Surgery. 3rd ed. Saint Louis, MO: Elsevier; 2010.

141. FitzGerald MP, Richter HE, Siddique S, Thompson P, Zyczynski $\mathrm{H}$, Ann Weber for the Pelvic Floor Disorders Network. Colpocleisis: a review. Int Urogynecol J Pelvic Floor Dysfunct. 2006;17(3):261-71.

142. Zebede S, Smith AL, Plowright LN, et al. Obliterative LeFort colpocleisis in a large group of elderly women. Obstet Gynecol. 2013;121(2 Pt 1):279-84.

143. Fitzgerald MP, Richter HE, Bradley CS, Ye W, Visco AC, Cundiff GW, et al. Pelvic support, pelvic symptoms, and patient satisfaction after colpocleisis. Int Urogynecol J Pelvic Floor Dysfunct. 2008;19(12):1603-9.

144. von Pechmann WS, Mutone M, Fyffe J, Hale DS. Total colpocleisis with high levator plication for the treatment of advanced pelvic organ prolapse. Am J Obstet Gynecol. 2003;189(1):121-6 
145. Hill AJ, Walters MD, Unger CA. Perioperative adverse events associated with colpocleisis for uterovaginal and posthysterectomy vaginal vault prolapse. Am J Obstet Gynecol. 2016;214(4):501.e1-6.

146. Denehy TR, Choe JY, Gregori CA, et al. Modified Le Fort partial colpocleisis with Kelly urethral plication and posterior colpoperineoplasty in the medically compromised elderly: a comparison with vaginal hysterectomy, anterior colporrhaphy, and posterior colpoperineoplasty. Am J Obstet Gynecol. 1995;173(6):1697-701.

147. Agarwala N, Hasiak N, Shade M. Graft interposition colpocleisis, perineorrhaphy, and tension-free sling for pelvic organ prolapse and stress urinary incontinence in elderly patients. J Minim Invasive Gynecol. 2007;14(6):740-5.

148. Dessie SG, Rosenblatt PL. Use of a vessel loop to ensure tunnel patency during LeFort colpocleisis. Int Urogynecol J. 2015;26(10):1541-3.

149. Koski ME, Chow D, Bedestani A, Togami JM, Chesson RR, Winters JC. Colpocleisis for advanced pelvic organ prolapse. Urology. 2012;80(3):542-6.

150. DeLancey JO, Morley GW. Total colpocleisis for vaginal eversion. Am J Obstet Gynecol. 1997;176(6):1228-32.

151. Petrikovets A, Fisher T, Krudy C, Sheyn D, Mangel J, Mahajan ST. LeFort colpocleisis: a step-by-step simulation video for female pelvic surgeons. Int Urogynecol J. 2018;29(6):917-9.

152. Constantin F, Veit-Rubin N, Ramyead L, et al. Rouhier's colpocleisis with concomitant vaginal hysterectomy: an instructive video for female pelvic surgeons. Int Urogynecol J. 2019;30(3):495-7.

153. Hanes CR 2nd. Vaginal sacral colpopexy: a natural orifice approach to a gold standard procedure. J Minim Invasive Gynecol. 2018;25(1):47-52.

154. Chen Y, Li J, Zhang Y, Hua K. Transvaginal single-port laparoscopy sacrocolpopexy. J Minim Invasive Gynecol. 2018;25(4): 585-8.

155. Liu J, Kohn J, Sun B, Guan Z, et al. Transvaginal natural orifice transluminal endoscopic surgery Sacrocolpopexy: tips and tricks. J Minim Invasive Gynecol. 2019;26(1):38-9.

156. Panel P, Soffray F, Roussillon E, et al. Glue mesh fixation: feasibility, tolerance and complication assessment. Results 24 months after laparoscopic sacrocolpopexy. J Gynecol Obstet Hum Reprod. 2017;46(4):333-8.
157. Lamblin G, Dubernard G, de Saint Hilaire P, et al. Assessment of synthetic glue for mesh attachment in laparoscopic sacrocolpopexy: a prospective multicenter pilot study. J Minim Invasive Gynecol. 2017;24(1):41-7.

158. Estrade JP, Gurriet B. Laparoscopic sacrocolpopexy with a vaginal prosthetic adhesive. J Minim Invasive Gynecol. 2015;22(6S):S67.

159. Liu J, Bardawil E, Lin Q, et al. Transvaginal natural orifice transluminal endoscopic surgery tubal reanastomosis: a novel route for tubal surgery. Fertil Steril. 2018;110(1):182.

160. Emmerson SJ, Gargett CE. Endometrial mesenchymal stem cells as a cell based therapy for pelvic organ prolapse. World J Stem Cells. 2016;8(5):202-15.

161. Glazener CM, Breeman S, Elders A, et al. Mesh, graft, or standard repair for women having primary transvaginal anterior or posterior compartment prolapse surgery: two parallel-group, multicentre, randomised, controlled trials (PROSPECT). Lancet. 2017;389(10067):381-92.

162. Sand PK, Koduri S, Lobel RW, Winkler HA, Tomezsko J, Culligan PJ, et al. Prospective randomized trial of polyglactin 910 mesh to prevent recurrence of cystoceles and rectoceles. Am J Obstet Gynecol. 2001;184(7):1357-62.

163. Grimes CL, Tan-Kim J, Whitcomb EL, Lukacz ES, Menefee SA. Long-term outcomes after native tissue vs. biological graftaugmented repair in the posterior compartment. Int Urogynecol J Pelvic Floor Dysfunct. 2012;23(5):597-604.

164. Marinaro F, Sanchez-Margallo FM, Alvarez V, Lopez E, Tarazona $\mathrm{R}$, Brun MV, et al. Meshes in a mess: mesenchymal stem cellbased therapies for soft tissue reinforcement. Acta Biomater. 2019;85:60-74.

165. FDA takes action to protect women's health, orders manufacturers of surgical mesh intended for transvaginal repair of pelvic organ prolapse to stop selling all devices. (2019) U.S. Food and Drug Administration. Available at: https://www.fda.gov/news-events/ press-announcements/fda-takes-action-protect-womens-healthorders-manufacturers-surgical-mesh-intended-transvaginal? fbclid=Iw A R 3 o W E ZrmF Q T trw N J T fP fR 6 Zn On TYGKJP6SnUlrblSHSiaqvqlkqCJUdgvs. Accessed October 25, 20192019

Publisher's note Springer Nature remains neutral with regard to jurisdictional claims in published maps and institutional affiliations. 